\title{
Thermal unmixing based downscaling for fine resolution diurnal land surface temperature analysis
}

\author{
Jiong Wang*, Oliver Schmitz, Meng Lu, Derek Karssenberg \\ Global Geo and Health Data Center, Utrecht University, Utrecht, the Netherlands \\ Department of Physical Geography, Faculty of Geosciences, Utrecht University, the Netherlands
}

\section{A R T I C L E I N F O}

\section{Keywords:}

Thermal downscaling

Spatiotemporal fusion

Remote sensing

MODIS

Landsat

LST

Unmixing

Diurnal

\begin{abstract}
A B S T R A C T
Due to the limitation in the availability of airborne imagery data that are high in both spatial and temporal resolution, land surface temperature (LST) dense in both space and time can only be obtained through downscaling of frequently acquired LST with coarse resolution. Many conventional downscaling techniques are only feasible in an ideal situation, where land surface factors as LST predictors are continuously available for downscaling the LST. These techniques are also applied only at large scales ignoring sub-regional variations. Based upon unmixing based approaches, this study presents an LST downscaling workflow, where only the coarse resolution of $1 \mathrm{~km}$ LST image at the prediction time is required. The conceptual backbone of the study is assuming that the LST patterns are governed by thermal behaviors of a fixed set of temperature sensitive land surface components. In operation, the study focuses on central Netherlands covering an area of $90 \times 90 \mathrm{~km}$. The MODIS and Landsat imagery acquired simultaneously are used as a coarse-fine resolution pair to derive downscaling mechanism which is then applied to coarse imagery at a time with missing fine resolution imagery. First, an optimal number of thermal components are extracted at fine resolution through the application of the non-negative matrix factorization (NMF). These components are assumed to possess unique temperature change patterns caused by combined effects of land cover change, radiance change, or both. Given the LST change and thermal components at coarse resolution, the LST change load of each component can then be obtained at the coarse resolution by solving a system of linear equations encoding thermal component-LST relationship. Such LST change load of thermal components is further unmixed to fine resolution and linearly weighted by the component distribution at fine resolution to obtain the fine resolution LST change. During the process, the coarse LST data is used directly without any resampling practice as shown in previous studies. Thus the technique is less time consuming even with a large downscaling factor of 30. The downscaled fine resolution LST represents an Rsquared of over 0.7 outperforming classic downscaling techniques. The downscaled LST differentiates temperature over major land types and captures both seasonal and diurnal LST dynamics.
\end{abstract}

\section{Introduction}

Land surface temperature (LST) is considered as a major descriptor of the thermal environment, which is continuously modified by changes of land use and land cover (LULC), and climate (Cai et al., 2018; Kalnay and Cai, 2003; Koc et al., 2018; Voogt and Oke, 2003; Weng et al., 2019). Our understanding of the ecological implications of thermal environments can be improved by exploring the LST in terms of its spatial and temporal patterns as well as the mechanisms behind these patterns (Anderson et al., 2008; Chen et al., 2006; Fu and Weng, 2016; Gallo et al., 1993; Gallo et al., 1995; Guo et al., 2015; Quattrochi and Luvall, 1999; Sandholt et al., 2002; Schwarz et al., 2012). Such spatial and temporal patterns can be highly dynamic as the LST is sensitive to land surface factors that are changing daily, seasonally, or yearly. Nevertheless, attempts have been made to utilize the LST to monitor LULC change and thermal conditions around, for instance, urban areas which are highly relevant to human settlement environment (Amiri et al., 2009).

LST information with high resolution in both space and time is favorable in several LST investigations, which revolves around three major themes (Li et al., 2013): (1) the patterns of the LST and the potential factors influencing the patterns (Rajasekar and Weng, 2009; Sobrino et al., 2012; Wu et al., 2014; Zhou et al., 2014), (2) the energy balance and fluxes at the land surface (Kuang et al., 2015; Kustas et al., 2016), and (3) the association between the LST and air temperature (Azevedo et al., 2016; Mira et al., 2017; Oyler et al., 2016). For

\footnotetext{
* Corresponding author at: Global Geo and Health Data Center, Utrecht University, Utrecht, the Netherlands.

E-mail addresses: j.wang2@uu.nl (J. Wang), o.schmitz@uu.nl (O. Schmitz), m.lu@uu.nl (M. Lu), d.karssenberg@uu.nl (D. Karssenberg).
} 
instance, fine resolution information enables detection of localized LST patterns such as the surface urban heat island (SUHI) (Shen et al., 2016a), or within-city LST variations (Mirzaei and Society, 2015; Pu et al., 2006). The influence of LULC types on LST is preferable at a high spatial resolution around $15 \mathrm{~m}$ (Connors et al., 2013). Recently, neighborhood health conditions are found to be partly explained by micro-scale LST patterns (Jenerette et al., 2016). Unfortunately, due to the resolution trade-off caused by the design of satellite sensors (Shen et al., 2016b), fine spatial resolution information is compromised by low temporal resolution (Kalma et al., 2008), which restricts continuous investigation of the abovementioned relationships. Many studies regarding surface energy balance (Li et al., 2008), evapotranspiration (Zhang et al., 2016), and LST-air temperature interaction (Stoll and Brazel, 1992; Voogt and Oke, 2003) are also restricted and only deployed at either low spatial or temporal resolution, leading to insufficient observation and contradictory conclusions (Coutts et al., 2016; Schwarz et al., 2012).

Thus, there is a need for LST information at a fine resolution in both space and time. Such information can be obtained through a downscaling scheme named spatiotemporal fusion. Different from super-resolution techniques in the image analysis community working on downscaling rules only within one pair of coarse-fine input image, spatiotemporal fusion attempts to explore the temporal change mechanism from multitemporal coarse resolution images and derive fine resolution change information to be added back to the input fine resolution at base time to obtain fine resolution predictions (Gao et al., 2006). Conventional spatiotemporal fusion techniques can be classified as (1) frequency transformation-based, (2) reconstruction-based, and (3) learning based (Chen et al., 2015; Tan et al., 2018), where reconstruction-based techniques can again boil down into sub-classes techniques of (a) weight-based, (b) regression-based and (c) unmixingbased sub-classes (Gevaert and García-Haro, 2015; Ma et al., 2018). Although originally developed for downscaling image reflectance acquired at the shortwave bands (Chen et al., 2015; Quan et al., 2018), when applied to downscaling thermal image radiance, the reconstruction-based techniques are considered as the primary choice (Huang et al., 2013; Weng et al., 2014), whereas the transformation-based and learning-based techniques are mainly applied to downscaling images at the shortwave bands. The regression-based technique explicitly requires land surface factors as predictors to capture the LST-land surface relationship and assumes that the relationship at coarse resolution can be applied to fine resolution land surface factors to obtain corresponding LST (Bechtel et al., 2012). In this sense, predictor datasets should be ideally available at each of the prediction time points to apply predictor-LST relationship to produce fine resolution LST at prediction time points, rendering a significant data demand and its limitation. Despite of improvements for the weight-based technique (Gao et al., 2006; Quan et al., 2018; Weng et al., 2014), the assumption of temporal change at each pixel location as weighted sum of spectrally similar neighboring pixels highlights the limitation in its application to places with heterogeneous LULC types. Recent attempts begin to rely on land surface change information such as vegetation change to recover the change residuals (Quan et al., 2018), some studies became in the form of combining weight-based and regression based concepts to improve downscaling results under the scheme of Geographically Weighted Regression (GWR) (Duan and Li, 2016; Peng et al., 2019). However, these studies largely relied on pixel level similarity to construct the weights. In the situation of downscaling LST, the varying moisture and vegetation with heterogeneous LULC types at the subpixel level would change radiance distinctively to spectrally similar pixels.

Instead of only focusing on spectral similarity at pixel level, the unmixing-based technique displays its strength by moving into subpixel level and attributing spectral change load to each of the subpixel primary land surface components named endmembers (Ma et al., 2018; Zhu et al., 2018). Utilizing the endmembers guarantees a solid mechanism to split subpixel temporal change information and avoid within-pixel-heterogeneity. Besides, the unmixing-based technique requires no extra land surface information as predictors at prediction time points and enhances its feasibility. However, one limitation of existing unmixing-based technique is that the adopted subpixel endmembers of substrate (mostly soil), vegetation and dark built surfaces (SVD) are only sufficient to encode pixel level reflectance as opposed to radiance. Another limitation lies in the assumption of this SVD unmixing-based technique that the temporal pixel level reflectance change is only due to reflectance change of the subpixel endmembers holding the endmember abundance unchanged. This assumption restricts the technique to the downscaling of images temporally adjacent to base time point with negligible endmember abundance change. The adopted SVD endmembers along with their assumed fixed abundance can easily be violated in downscaling images with large temporal gap and radiance variation in thermal images. The thermally sensitive components such as vegetation and moisture can change in both radiance and abundance in a short period of time. Built upon the concept of SVD unmixing-based technique, this study considers the combined change of radiance and abundance of land surface endmembers in downscaling of thermal images to obtain temporally dense LST data with fine spatial resolution. The study is presented in a comparison manner using the classic weightbased along with the existing SVD unmixing-based technique as benchmarks. The major aims of this study can be summarized as to (1) improving the unmixing-based downscaling technique in the application to thermal data, (2) testing the performance of the proposed subpixel level characterization of thermal patterns through comparison to existing techniques, and (3) envisioning the applicability of the proposed technique in city scale study area and over different LULC types. The benchmark and proposed techniques are presented first in methodology, followed by performance comparison in results. The performance of the proposed technique is further presented by examining the LST patterns over different LULC types, which directs the way of applying the proposed technique in environment and microclimate studies. The study closes by discussing the proposed technique in the context of current technical and methodological limitations in thermal downscaling.

\section{Methodology}

The key assumptions of this study could be summarized at two levels: (1) the generalized scenario where both of subpixel endmember radiance and abundance, as opposed to only the radiance, are changing, and (2) the combined change of subpixel endmember radiance and abundance follows unique pattern with given composition of LULC, leading to the assumption of subpixel thermal components possessing discriminative thermal behaviors. Thus, the first step is to extract thermal components. The extraction is followed by splitting the coarse resolution LST change to fine resolution according to the distribution of thermal components at fine resolution. The performance of the proposed technique is to be compared with benchmark conventional techniques. This study is based in the central Netherlands with diverse LULC types so that performances of the techniques can be examined across different land types.

\subsection{Study area and data}

The study area in the Netherlands spans the province of Utrecht, Gelderland and North Brabant involving various LULC types forming a $90 \times 90 \mathrm{~km}$ region (Fig. 1(a)). Major cities such as Utrecht, Nijmegen and Eindhoven are included. Besides cities, forests and few bare lands, the agricultural activities in the Kromme Rijn area and along the Lek river would test the applicability of the proposed technique across diverse land types. Fig. 1(b) shows that the sample fine resolution LST product of the study area distributed with a pixel size of $30 \mathrm{~m}$ captured by the Landsat- 8 TIRS. In the figure, the temperature variations within urban, across urban and rural, and between bareland and vegetated 
(a)

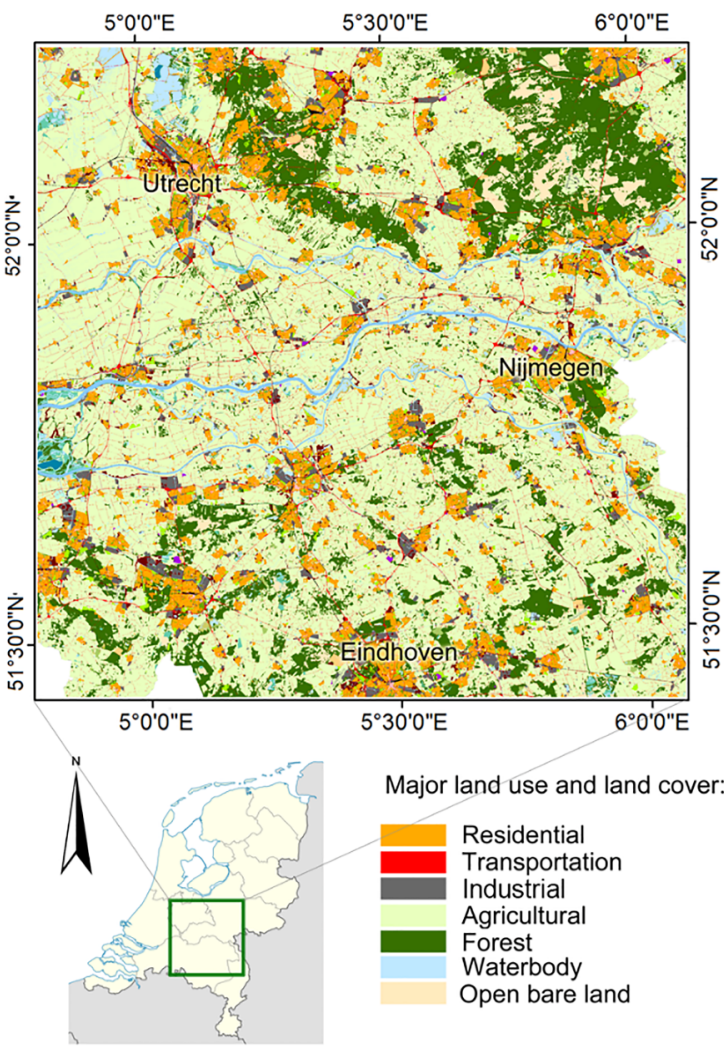

(b)
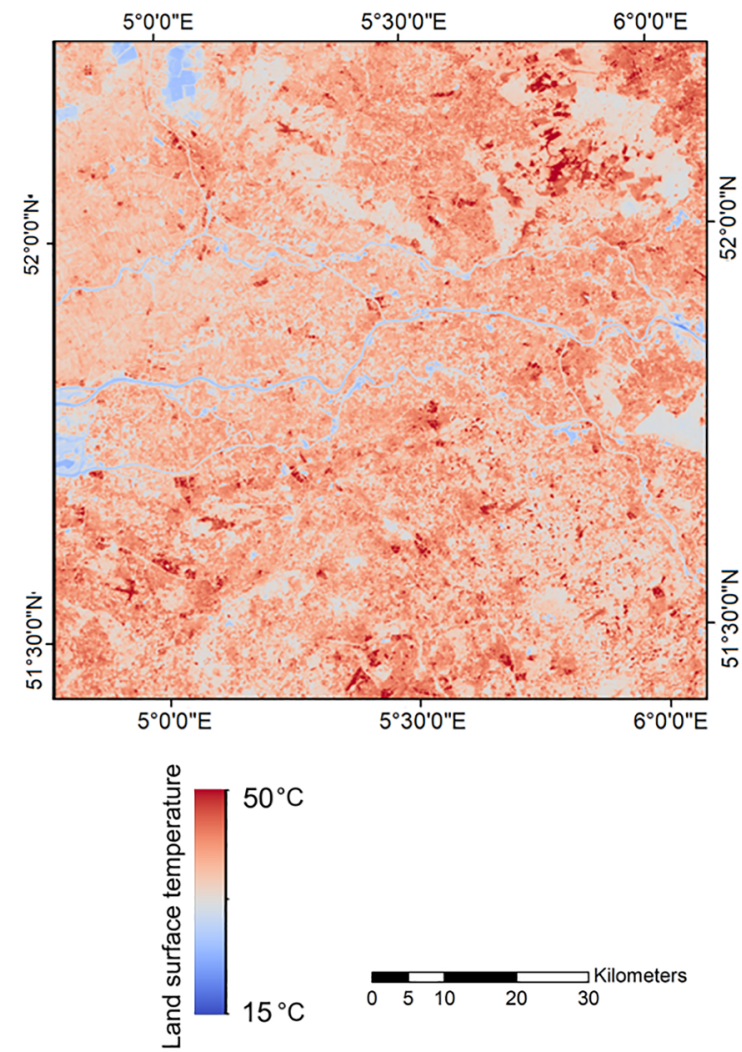

Fig. 1. (a) The LULC map in the study area and (b) sample land surface temperature (LST) snapshot acquired at $10: 32$ on July 26 th, 2018.

area are already visible.

The proposed thermal component (TC) unmixing-based downscaling technique is applied by using coarse-fine image pairs consisting of the MODerate-resolution Imaging Spectroradiometer (MODIS) LST/E Daily $1 \mathrm{~km}$ Grid products and the Landsat-8 Level- 1 Thermal Infrared Sensor (TIRS) products distributed at the resolution of $30 \mathrm{~m}$. Although the TIRS data is of $100 \mathrm{~m}$ original resolution, the data has been resampled through cubic convolution to match the other bands distributed at $30 \mathrm{~m}$ (Cho et al., 2018). In order to avoid introducing further uncertainty in aggregating the data back to $100 \mathrm{~m}$ as cubic convolution is irreversible, we attempted to provide convenience for users directly work on data of $30 \mathrm{~m}$ resolution and stay with the distributed resolution. The Landsat-8 thermal imagery with a fine spatial resolution of $30 \mathrm{~m}$ is only acquired every 16 days at around 10:32 local time (Jiménez-Muñoz et al., 2014), where the LST can be derived by applying the classic mono-window algorithm to band 10 due to its smaller calibration uncertainty compared to band 11 (Barsi et al., 2014; Wang et al., 2015). In contrast, the frequently acquired MODIS/Terra (MOD11A1) and MODIS/Aqua (MYD11A1) V6 datasets at $1 \mathrm{~km}$ resolution provide LST measurements 4 times per day (Wan, 2014). Specifically, the MOD11A1 contains LST at 10:30 and 22:30 local time (24-hour clock), while the MYD11A1 is acquired at 01:30 and 13:30 local time. Furthermore, both radiance and temperature based validations of the MODIS V6 thermal products indicated a promising accuracy (RMSE less than $1.3 \mathrm{~K}$ ) in most cases (Duan et al., 2019; Duan et al., 2018). This temporal frequency is attractive to be downscaled for examining diurnal and daily LST variation at fine spatial resolution.

During experiment, a pair of coarse-fine LST imagery of the MOD11A1 and Landsat-8 TIRS with least cloud contamination at the base time in the morning of July 26, 2018 (Fig. 2(a) and (b)) has been used as the input image pair for downscaling the remaining MODIS LST data over a summer period in 2018 containing heatwaves (Beniston et al., 2004; Chase et al., 2006; Rebetez et al., 2009). In this way, the downscaling technique is expected to show not only fine resolution spatial variation but also temporal anomaly of the LST during the heatwaves. Although there can be a few minutes gap between the acquisition time of the input pair of Landsat-8 and MOD11A1 imagery (Table 1), the LST change over few minutes is considered to be negligible. With the Landsat distributed resolutions of $30 \mathrm{~m}$ and MODIS data with resolution of $1 \mathrm{~km}$, this study has been working on a downscaling task with a factor of $>30$.

The proposed TC unmixing-based technique required information about subpixel thermal components similar as the subpixel endmember information demanded by the SVD unmixing-based technique. Yet the thermal components were unknown contrasted with the already available SVD endmembers reflectance. To extract temporally stable information regarding the thermal components, multi-temporal bands including those at the base time have been involved (Table 1). All the techniques involved for comparison in this study required no extra information other than the coarse LST at the prediction time point, setting the comparison consistent.

To validate the downscaling results, two prediction time points spanning over approximately one year were identified on May 7th, 2018 and May 26th, 2017, where Landsat fine resolution LST was available for verifying predicted fine resolution LST during both night and day (Fig. 2(c)). The used data is shown in Table 1. Atmospheric correction was applied to the data before the following processes. The atmospheric profile parameters adopted were obtained from the NASA Atmospheric Correction Parameter Calculator (https://atmcorr.gsfc. nasa.gov/).

\subsection{From the classic weight-based to unmixing-based technique}

As the downscaling techniques have been implemented at both reflectance/radiance and LST level (Weng et al., 2014), the following content sticks to the convention of using radiance or reflectance, and 
(a)

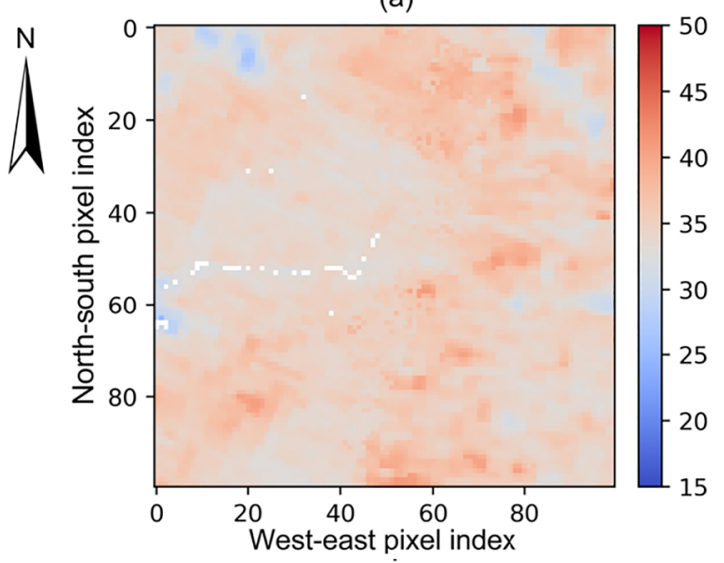

(b)

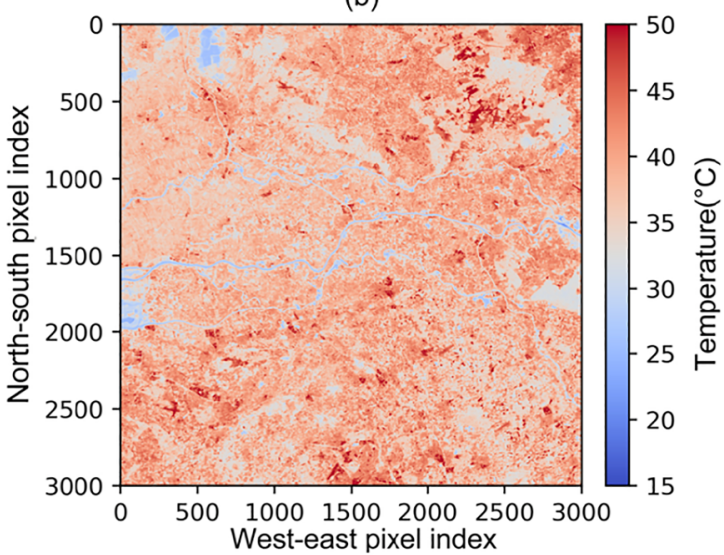

(c)

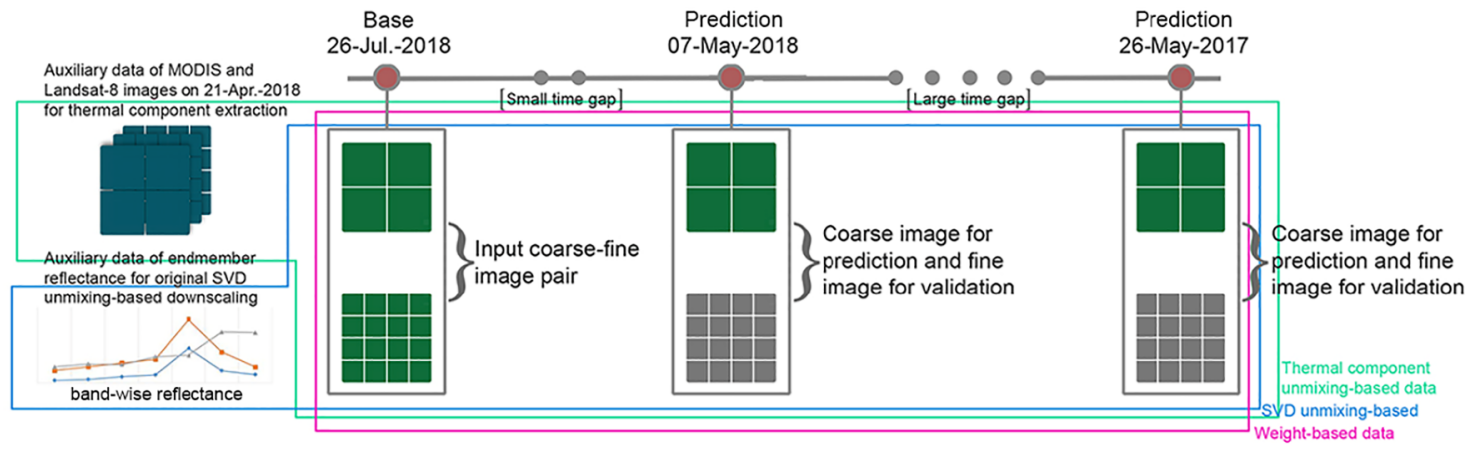

Fig. 2. Setup of data and methods: (a) MOD11A1 and (b) the Landsat-8 images at base time of July 26th, 2018 used as the input coarse-fine resolution image pair for downscaling MODIS LST data. (c) Data and downscaling techniques involved in the study.

Table 1

Data involved in this study.

\begin{tabular}{|c|c|c|c|c|c|}
\hline Date & MODIS overpass time & MODIS bands & Landsat overpass time & Landsat bands & Purpose \\
\hline 26-Jul.-2018 & $22: 36$ & MOD11A1 band 1 & $10: 32$ & LC08 band 4, 5, 10 & Thermal component extraction \\
\hline 21-Apr.-2018 & $22: 36$ & MOD11A1 band 1 & $10: 33$ & LC08 band 4, 5, 10 & Thermal component extraction \\
\hline 26-Jul.-2018 & $10: 30$ & MOD11A1 LST & $10: 32$ & LC08 LST & Coarse and fine input pair \\
\hline 07-May-2018 & $10: 30$ & MOD11A1 LST & $10: 32$ & LC08 LST & Prediction and validation pair \\
\hline 26-May-2017 & $22: 36$ & MOD11A1 LST & 21:07 & LC08 LST & Prediction and validation pair \\
\hline
\end{tabular}

starts with a brief recap presenting the transition from weight-based to unmixing-based technique.

The weight-based downscaling technique focuses on the land surface reflectance $R$. The downscaling starts with resampling the coarse input image to the fine resolution at the base time point $t_{b}$ (Gao et al., 2006). With the same pixel size, the temporal change of coarse resolution information $R_{c}$ from $t_{b}$ to prediction time point $t_{p}$ at each pixel location is added to the base time input fine resolution reflectance $R_{f}$ at the corresponding pixel location so that the fine resolution at $t_{p}$ is obtained. Since the resampled coarse reflectance can be quite different from the fine resolution information at each of the fine pixel locations, the predicted reflectance $R_{f}$ of any target fine resolution pixel located at $(i, j)$ on an image is a $W_{k l}$ weighted prediction at several similar pixel locations $(k, l)$ in the neighborhood window $w$ of the target prediction. The prediction is given by (Gao et al., 2006):

$R_{f}\left[i, j, t_{p}\right]=\sum_{k}^{w} \sum_{l}^{w} W_{k l} \times\left(R_{f}\left[i_{k}, j_{l}, t_{b}\right]+R_{c}\left[i_{k}, j_{l}, t_{p}\right]-R_{c}\left[i_{k}, j_{l}, t_{b}\right]\right)$

However, the assumption of consistent change of reflectance among similar pixels is only valid when LULC within the pixels is homogeneous. The SVD unmixing-based technique addresses this by considering LULC heterogeneity at subpixel level (Ma et al., 2018). The LULC heterogeneity is encoded by using substrate (mostly soil), vegetation and dark built surfaces as fundamental subpixel endmembers contributing to pixel level reflectance. Thus the fine resolution reflectance $R_{f}$ is further split into a linear combination of land surface endmember reflectance $E$ weighted by endmember abundance $A$ plus the residual $\varepsilon$ if $m$ endmembers are assumed:

$R_{f}\left[i, j, t_{b}\right]=\sum_{m} A_{f}\left[i, j, m, t_{b}\right] \times E_{f}\left[m, t_{b}\right]+\varepsilon\left[i, j, t_{b}\right]$

If the abundance $A$ and residual $\varepsilon$ remain stable from $t_{b}$ to $t_{p}$, the $\varepsilon$ is cancelled in calculating the change of reflectance $\Delta R_{f}$. And the fine resolution prediction in Eq. (1) is determined solely by change of reflectance and becomes:

$R_{f}\left[i, j, t_{p}\right]=R_{f}\left[i, j, t_{b}\right]+\Delta R_{f}=R_{f}\left[i, j, t_{b}\right]+\sum_{m} A_{f}[i, j, m] \times \Delta E_{f}[m]$

The major different between Eqs. (3) and (1) is the subpixel level information is considered to take care of heterogeneity. With known information of SVD endmember reflectance (Small, 2003; Small, 2004), the SVD unmixing-based technique follows a three-step workflow to (1) use spectral unmixing to obtain endmember abundance $A$ through Eq. 
(2), (2) use spatial unmixing to obtain fine resolution endmember reflectance change $\Delta E$ from temporal change reflectance change at coarse resolution, and (3) use linear mixture to combine endmember reflectance change weighted by the endmember abundance to obtain fine pixel level reflectance change, which, as shown in Eq. (3), can be added to the base time reflectance to make prediction (Ma et al., 2018).

\subsection{Introducing thermal components for unmixing-based technique}

This study detected a major defects of existing SVD endmembers along with their fixed abundance in characterizing thermal regulations. At each pixel on a thermal image, the reflectance becomes radiance Rad, and the assumption of SVD endmembers with unchanged abundance is insufficient to capture the Rad change mechanism. Other endmembers governing the land surface energy balance such as surface moisture can modify evaporation and evapotranspiration with either a change in the endmember abundance or radiation. Thus, the change of abundance $\Delta A$ needs also to be introduced to Eq. (3) as an unknown term. Instead of tracking down the change sources of Rad in terms of endmember $A$ and $E$, an alternative is to assume the existence of a fixed number $m$ of subpixel level thermal components that possess unique and combined change patterns of both $A$ and $E$ without explicitly differentiating between the two. Intuitively, any continuous patch of relatively uniform LULC type, such as any forest species, high rise residential, low rise residential or crop species can potentially be considered as a fraction of subpixel thermal components, within which distinctive combined change pattern of endmember radiance and abundance is uniform. Then the change of Rad similar to the change of reflectance $\Delta R$ in Eq. (3) can be expressed as thermal components radiance change weighted by their fractions $F$ :

$\Delta \operatorname{Rad}[i, j]=\sum_{m} F[i, j, m] \times g(\Delta A, \Delta E, m)=\sum_{m} F[i, j, m] \times \Delta Q[m]$

where the abundance change $\Delta A$ and radiance change $\Delta E$ are treated in combination within a black box denoted as function $g$ and further as $\Delta Q$. Although the exact change amount of the abundance and endmember radiance is unknown, they, in combination should produce a finite number of specific change patterns as LULC types are finite. In this way, the unknown terms are integrated and reduced one in the form of Eq. (3), which solves can be solved given the coarse temporal change of radiance $\Delta R a d$. The above assumption of thermal component (TC) forms the technical backbone of the proposed TC unmixing-based downscaling technique. The details of implementation are shown as below.

\subsection{Implementing the TC unmixing-based technique}

As the proposed TC unmixing-based technique denies the application of existing spectral library encoding endmember reflectance of SVD, the first step similar to spectral unmixing mentioned in Section 2.2 is to obtain information of the TCs equivalent to endmember abundance in SVD unmixing-based technique. While the SVD depends on spectral library to query endmember reflectance, no prior knowledge of the TC spectral is available for obtaining the fraction of the TC.

Without already available spectral library of the TC, external information could be obtained through multiple bands given the TC fraction was assumed to be temporally stable according to Eq. (4). Extracting stable components from multiple bands directs to the application of the non-negative matrix factorization (NMF). The NMF treats target features such as images as a linear combination of a fixed number of base components and weights, where both components and weights are non-negative (Lee and Seung, 2001). Such non-negative property is preferable for obtaining meaningful information from remote sensing based observations.

In operation, multiple thermally sensitive bands were included as auxiliary data (Fig. 2(c)). Each band was assumed to be a combined response of thermally sensitive components, which are the TCs. Since fractions of the TCs were assumed to be temporally stable, multiple bands across time would force the NMF to seek TCs that could be combined to produce multi-temporal bands. Thus thermally meaningful bands such as near and thermal infrared bands across time were considered to include sufficient information about moisture and vegetation (Fensholt and Sandholt, 2003; Tucker, 1980). Involved bands were the Landsat- 8 band 4, 5, 10 on both July 26 and April 21, 2018. Besides, the cubic convolution resampled MODIS night images from the two days were also used to encode daily thermal dynamics (Table 1 ). All of the 8 bands are of size $n=3000 \times 3000$ pixels and were stacked as the input to the NMF encoding seasonal and diurnal thermal dynamics. The input is now a radiance matrix $\boldsymbol{R a d}$ with $n=3000 \times 3000$ rows and $m=8$ columns, which is decomposed through the NMF as:

$\boldsymbol{R a d}_{n \times m}=\boldsymbol{F}_{n \times r} \cdot \boldsymbol{P}_{r \times m}$

where $r$ is the number of the TCs can be determined by the NMF itself by examining the explained variance and residuals. In this sense, the NMF is capable to determine the non-negative TCs and their number at the same time. Although, mathematically, the NMF is simple as a product of two matrices, the interpretation can be flexible in applications. In image analysis (Lee and Seung, 1999), $\boldsymbol{F}$ can be treated as $r$ base images with the same size $n$ of the input images, while $\boldsymbol{P}$ encode $m$ columns of the weights for combining the base images for each of the $m$ input images. In spectral analysis (Miao and Qi, 2007), $\boldsymbol{F}$ can be the fraction map of $r$ primary components, and $\boldsymbol{P}$ encodes the response of the components at each of the $m$ input bands.

Given any $r$, a positive solution of both $\boldsymbol{F}$ and $\boldsymbol{P}$ can be obtained by minimizing the unexplained or residual variance expressed as:

$\min _{\boldsymbol{F}, \boldsymbol{P}}\|\boldsymbol{R a d}-\boldsymbol{F} \cdot \boldsymbol{P}\|\{\boldsymbol{F} \geq 0, \boldsymbol{P} \geq 0\}$

The solution can be derived through several iterative algorithms, among which the multiplicative update rule is the most popular approach (Lee and Seung, 2001).

Holding the temporally stable $\boldsymbol{F}$ at fine resolution, Eq. (4) at fine resolution becomes:

$$
\begin{aligned}
\Delta \operatorname{Rad}_{f}[i, j] & =\sum_{m} F_{f}[i, j, m] \times g\left(\Delta A_{f}, \Delta E_{f}, m\right) \\
& =\sum_{m} F_{f}[i, j, m] \times \Delta Q_{f}[m]
\end{aligned}
$$

where $\Delta Q_{f}$ needs to be provided to obtain $\Delta R a d_{f}$. Although $\Delta Q_{f}$ is not available at fine resolution, its coarse resolution counterpart can be obtained as the coarse resolution $F_{c}$ can be obtained through simple pixel aggregation by averaging (Ma et al., 2018), while the coarse resolution temporal change of radiance $\Delta \operatorname{Rad}_{c}$ can obtained through:

$\Delta \operatorname{Rad}_{c}[i, j]=\operatorname{Rad}_{c}\left[i, j, t_{p}\right]-\operatorname{Rad}_{c}\left[i, j, t_{b}\right]$

Now the spatial unmixing can be applied at coarse resolution to obtain $\Delta Q_{c}$ through:

$\Delta \operatorname{Rad}_{c}[i, j]=\sum_{m} F_{c}[i, j, m] \times \Delta Q_{c}[m]$

During calculation, similar to the original SVD unmixing-based experiment (Ma et al., 2018), pixels in the $3 \times 3$ neighborhood of the target pixel are included to form a system of linear equations so that $m$ unknow $\Delta Q_{c}$ can be solved.

To derive the fine resolution $\Delta Q_{f}$, the sensor difference should be considered as the observation time, atmospheric correction and radiation anisotropy between the acquisition of the coarse-fine imagery would cause systematic discrepancy (Steven et al., 2003). A handy approach is to use a linear model to capture the coarse-fine resolution sensor relationship (Shen et al., 2013), where fine resolution data is linearly correlated to coarse resolution data through coefficient $\alpha$ and bias $\beta$ : 


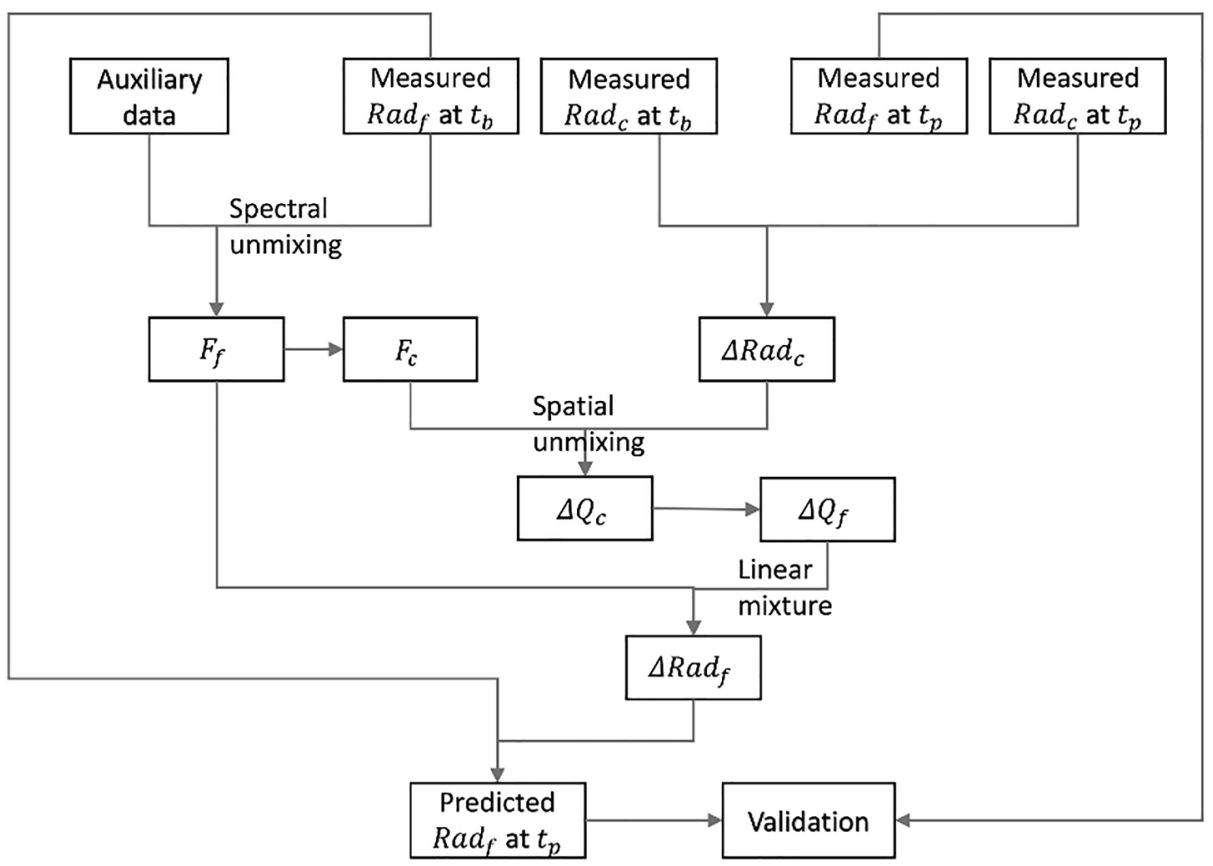

Fig. 3. Workflow of the TC unmixing-based downscaling technique.

$Q_{c}=\alpha Q_{f}+\beta$

Then, the temporal difference $\Delta Q_{c}$ derived from Eq. (10) is:

$\Delta Q_{c}=\alpha \Delta Q_{f}$

where $\beta$ is cancelled. The obtained $\Delta Q_{f}$ at the central coarse pixel can be assigned to corresponding fine resolution pixels within the extent of this coarse pixel (Gevaert and García-Haro, 2015; Ma et al., 2018), and the linear mixture can be applied to obtain the fine resolution temporal radiance change through:

$\Delta \operatorname{Rad}_{f}[i, j]=\sum_{m} F_{f}[i, j, m] \times \Delta Q_{f}[m]$

where the $\Delta \operatorname{Rad}_{f}$ can be added back to the base time radiance to make the final prediction as:

$\operatorname{Rad}_{f}\left[i, j, t_{p}\right]=\operatorname{Rad}_{f}\left[i, j, t_{b}\right]+\Delta \operatorname{Rad}_{f}[i, j]$

If the downscaling technique is implemented at the radiance level as opposed to the LST level, the radiance can be transformed into LST by applying Planck's law (Weng et al., 2014). The entire process can be summarized as a workflow shown in Fig. 3.

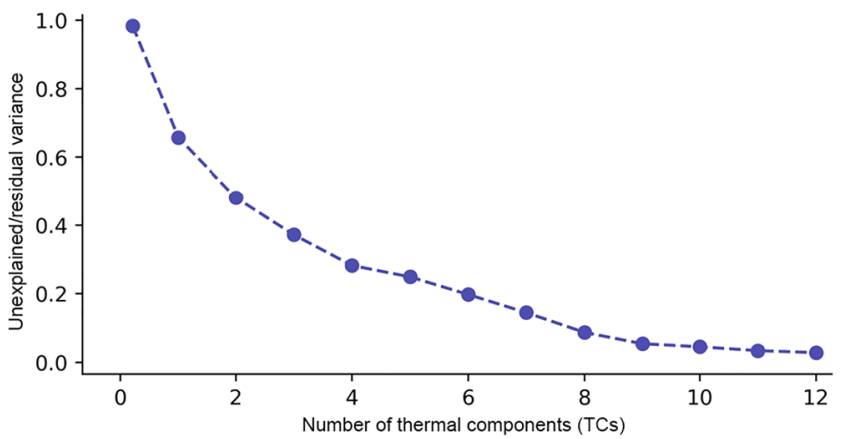

Fig. 4. Increasing number of thermal components along with decreasing residual variance by applying the NMF.

\section{Results}

\subsection{Thermal components}

Iterative application of Eq. (6) shows that the temporal dynamics of radiance encoded in the 8-band input data can be fully interpreted by using 8 or more TCs. In contrast, using only 1 TC only explains less than $40 \%$ of the information leaving over $60 \%$ of the information unexplained (Fig. 4). However, the TCs as primary components would be meaningful if the number of the TCs is less than that of the input bands. Two criteria are used to determine the optimal number of TCs: (1) the number should be less than the NMF features in the original 8-band dataset, which is 8 , and (2) each TC should explain a considerable amount of information in the original dataset. The criteria lead to choosing 4 TCs. Adding more TCs only brings less than 5\% residual variance decrease and the approximation residual levels off.

The TC fraction maps $\boldsymbol{F}$ produced by applying Eq. (6) are visualized in Fig. 5. Each pixel of the study area is split into subpixel fractions of 4 TCs possessing unique LST change patterns. Along with the spatial configuration of the TCs, the spectral response curves of the TCs are also provided in Fig. 6 to physically justify the thermal characteristics of the TCs.

Among all of the 4 TCs, the patterns of TC1 and TC2 are both visually prominent and spectrally stable. TC1 possesses a typical spectral pattern of vegetated land surface with the highest thermal response at band 10 of Landsat-8 TIRS in both April and July, as well as its strong response at near infrared wavelength at band 5 with a drop at around the visible red wavelength at band 4 (Fig. 6) (Estoque and Murayama, 2015). The spectral pattern is quite stable with a slight increase of thermal response from spring to summer, yet with a decrease of vegetation cover according to the change of its responses in band 4 and 5 during the same time span. Such "vegetated" thermal pattern can be spatially examined in Fig. 5. TC1 is mainly located around suburban area, where the fraction of this component appears to be high especially in seasonal irrigated farmland with seasonal vegetation and moisture change as shown by the zoom-in scene. Nevertheless, there are also distributions of TC1 in forest area, which highlights the fact that the property of TCs is associated with LULC. Here, similar LULC may share similar thermal patterns which are captured by $\mathrm{TC} 1$, however, forest 
(a)

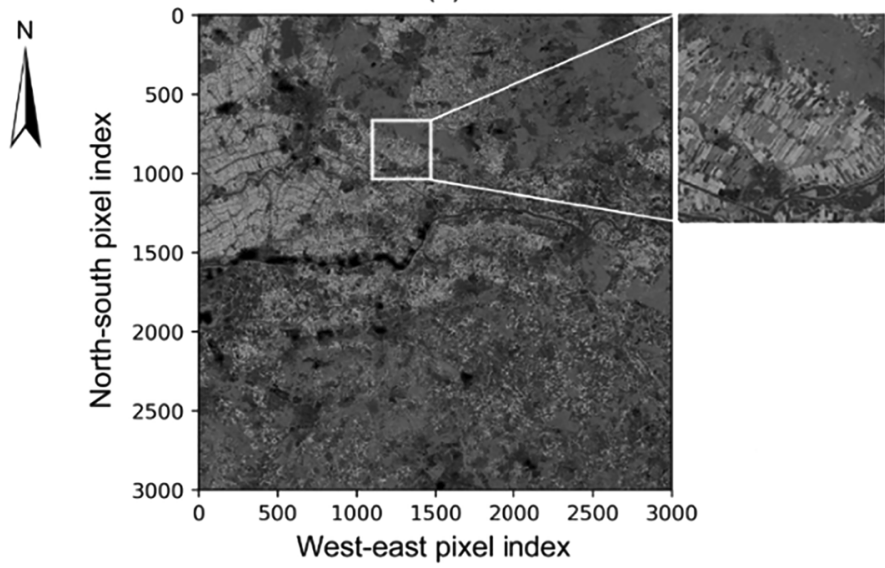

(c)

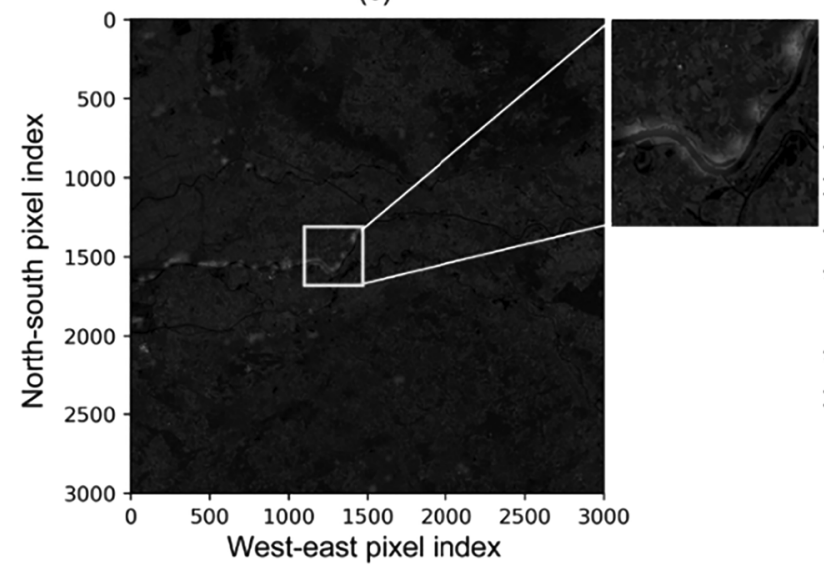

(b)

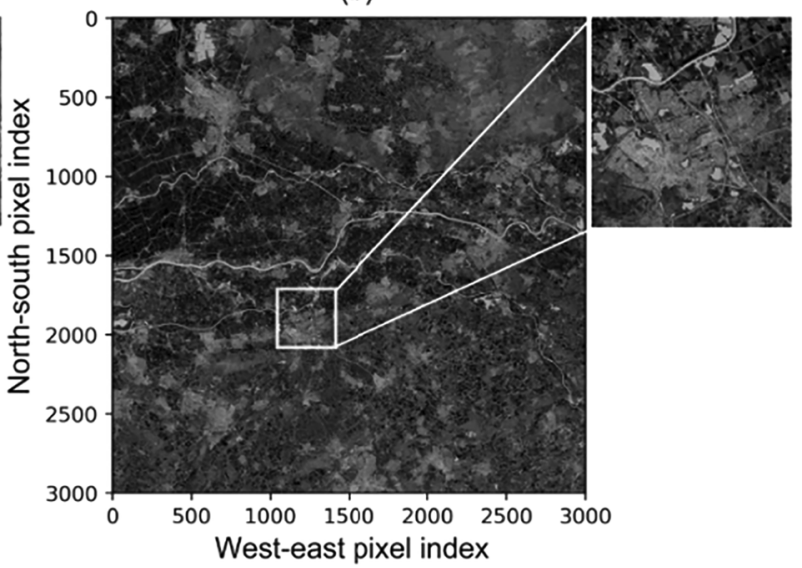

(d)

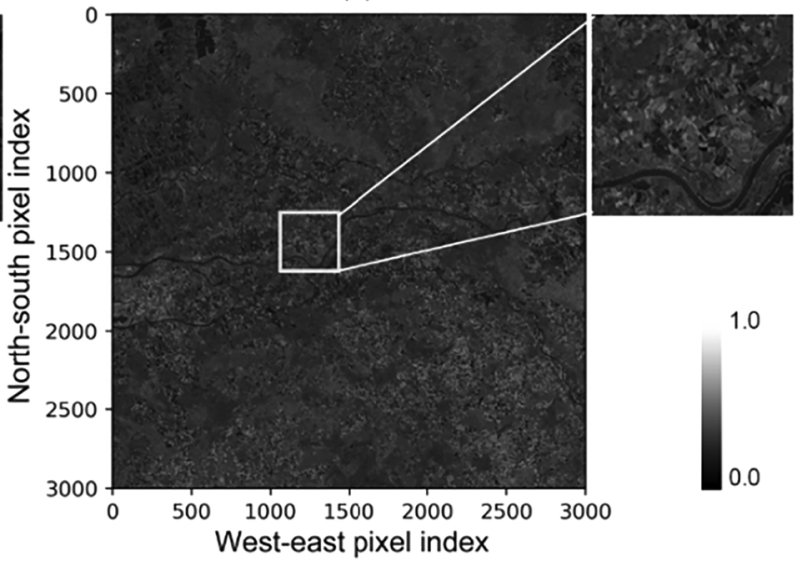

Fig. 5. Fraction maps of the 4 TCs in (a), (b), (c) and (d) with distinguishable spatial distribution patterns.

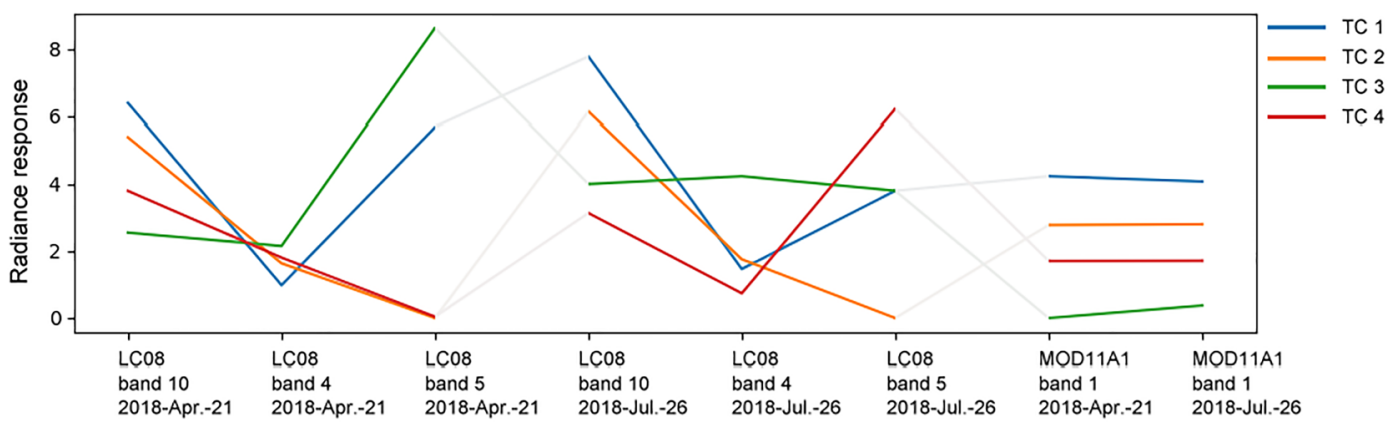

Fig. 6. Spectral response of TCs at the bands of the input data.

and crop land are not exactly identical in thermal behavior and thus differentiated by the fractions of TC1.

The spectral response of TC2 shows a high thermal response at band 10 of Landsat- 8 TIRS in both April and July, yet the responses at both visible red wavelength and infrared level off (Fig. 6). At the same time, the nighttime thermal response also stays higher than those of TC3 and TC4. The above patterns imply a low vegetated area with large specific heat capacity, which means the component absorbs radiation and mains temperature till night (Estoque and Murayama, 2015). In conjunction with the spatial distribution of TC2, it is not surprise to recognize most of this component are found around cities, as well as rivers without vegetation. Such pattern can be further justified as a combined spectral pattern of built areas and waterbodies that remain warm and poorly vegetated. Although TC2 is also found in some bare land and in-land water bodies, the spatial and spectral patterns of TC2 again confirm the shared thermal patterns captured by the extracted TC.

TC3 represents a spectral response of vegetated area in April with high response around near infrared wavelength at band 5 of Landsat-8 data. In contrast, during summer time in July, the component displays a spectral pattern of low vegetated area with low thermal response. The distinctive patterns in two season indicate sharp and unreasonable moisture and vegetation changes. The spatial pattern in Fig. 5(c) provides a complement to the spectral patterns by locating TC3 mainly along riverbanks, typically floodplains in the context of the Netherlands, where sharp vegetation and moisture change are reasonable. TC4 represents typical spectral response of waterbodies in April, and vegetated areas in July. The spatial distribution in Fig. 5(d) indicates that TC4 mainly falls into farmland scattered among areas with TC1. One possible interpretation is that TC4 can be farmland yet may not be seasonally irrigated since it is getting dryer with, though, large increase 
Table 2

TC radiance response at each of the 8 input bands.

\begin{tabular}{|c|c|c|c|c|c|c|c|c|}
\hline \multirow{2}{*}{$\begin{array}{l}\text { Date } \\
\text { Band }\end{array}$} & \multicolumn{3}{|c|}{ 21-Apr.-2018 } & \multicolumn{3}{|c|}{ 26-Jul.-2018 } & \multirow{2}{*}{$\begin{array}{l}\text { 21-Apr.- } \\
2018 \\
\text { MOD11A1 } \\
\text { band 1 }\end{array}$} & \multirow{2}{*}{$\begin{array}{l}\text { 26-Jul.- } \\
\text { 2018 } \\
\text { MOD11A1 } \\
\text { band 1 }\end{array}$} \\
\hline & $\begin{array}{l}\text { LC08 } \\
\text { band } \\
10\end{array}$ & $\begin{array}{l}\text { LC08 } \\
\text { band } \\
4\end{array}$ & $\begin{array}{l}\text { LC08 } \\
\text { band } \\
5\end{array}$ & $\begin{array}{l}\text { LC08 } \\
\text { band } \\
10\end{array}$ & $\begin{array}{l}\text { LC08 } \\
\text { band } \\
4\end{array}$ & $\begin{array}{l}\text { LC08 } \\
\text { band } \\
5\end{array}$ & & \\
\hline TC1 & 6.40 & 0.98 & 5.70 & 7.79 & 1.47 & 3.81 & 4.24 & 4.09 \\
\hline TC2 & 5.40 & 1.64 & 0.00 & 6.15 & 1.76 & 0.00 & 2.78 & 2.81 \\
\hline TC3 & 2.56 & 2.16 & 8.64 & 4.01 & 4.24 & 3.81 & 0.00 & 0.38 \\
\hline TC4 & 3.80 & 1.81 & 0.04 & 3.14 & 0.74 & 6.23 & 1.71 & 1.72 \\
\hline
\end{tabular}

(a)
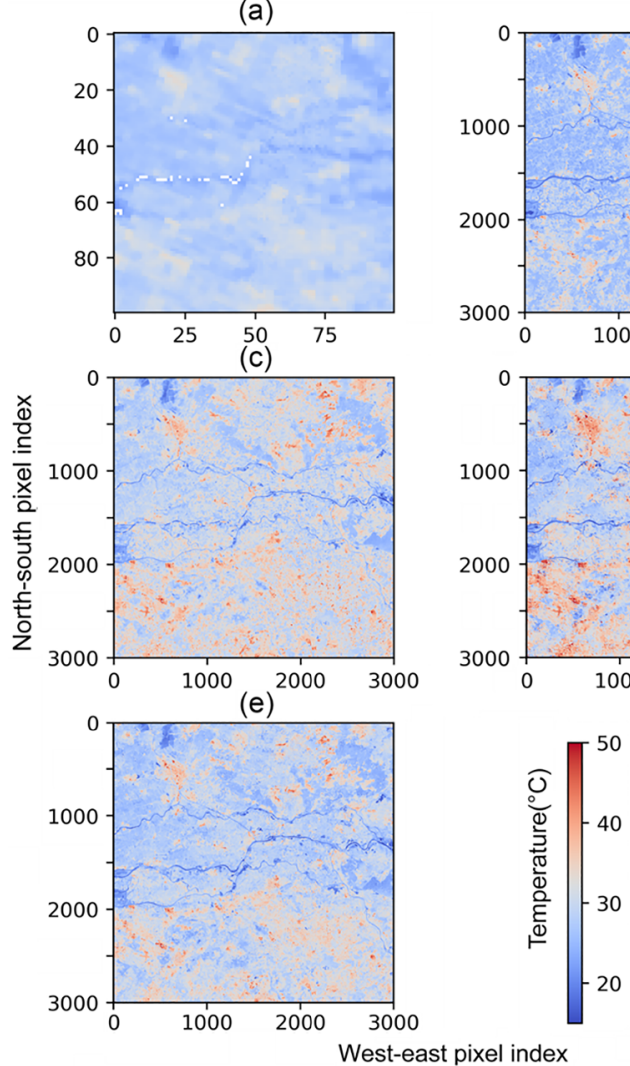

Fig. 7. Spatial pattern of daytime predictions using weight-based, SVD unmixing-based, and TC unmixing-based techniques. The coarse resolution LST on May 7th, 2018, for predicting the fine resolution LST is shown in the (a), while the validation LST is in (b). The downscaled LSTs using weight-based, SVD unmixing-based and TC unmixing-based techniques are shown in (c), (d) and (e), respectively.

of vegetation cover and only a minimal increase in the thermal band.

Now, the NMF has extracted 4 types of TCs reasonably distributed in certain LULC with distinctive thermal patterns. More detailed values of the spectral responses of the TCs are provided in Table 2.

\subsection{Visualizing daytime prediction}

The predictive capacity of the TC unmixing-based downscaling technique on daytime LST is compared with classic weight-based and SVD unmixing-based techniques (Fig. 7). Note the pixels on the base time fine resolution image located at those with missing values on the coarse resolution image at prediction time are not masked out for visual convenience, thus the downscaled $\Delta R a d_{f}$ obtained from Eq. (13) with missing values are filled with base time fine resolution pixels. For statistical analysis and validation, these pixels located at places with missing values are excluded. Visually, all the three technique more or less overestimate the high temperature pixels and underestimate the low temperature pixels in the study area, which bring larger variance to the predictions compared to the validation data (Fig. 7(b)). Compared to the TC unmixing-based technique, the overestimations caused by the weight-based and SVD unmixing-based techniques are especially prominent around built-up areas and among the potentially dry farmland by referring back to Fig. 1(a). Specifically, the overestimation of weight-based technique in high temperature areas around cities appears to be similar to the performance of the TC unmixing-based technique. However, the overestimation caused by the weight-based technique pertains not only to high temperature area, but also to areas around the average temperature of the study area. This can be observed by comparing Fig. 7(b) and (c), and scrutinizing how pixels in blue to the south and east to the city of Utrecht are predicted to be in pink. The overestimation is exaggerated by the SVD unmixing-based technique. The cities and residential clusters are especially overestimated, while large parts of the farmland to the southeast of the study area are predicted to be over $40{ }^{\circ} \mathrm{C}$. One possible interpretation is that the adopted SVD endmembers are not capable to capture the pixel level thermal patterns and improperly split the temperature change into pixels dominated by any one of the endmembers.

\subsection{Statistics of daytime prediction}

Due to the visually distinguishable performances among all the three techniques as well as the over and underestimation across the study area within each of the techniques, the statistical analysis is deployed to divide the performances of all the techniques over the entire study area into performances over major LULC types.

Over the entire study area, the performances of all three techniques shown in Fig. 8 are consistent with the visual patterns observed in Fig. 7. The variances in the predictions caused by over and underestimation are instantly visible through the spread of the scatterplots, where SVD unmixing-based brings the largest over and underestimation to the prediction due to improper split of thermal change by the SVD endmembers. The proposed TC unmixing-based technique produces the least amount of variance in the prediction, while the performance of the weight-based technique stands between the other two techniques. Specifically, the weight-based technique performs similarly to the proposed TC unmixing-based technique especially around places with higher temperature such as built-up areas. The shapes at the high temperature tails of the scatterplots in Fig. 8(a) and (c) are almost identical. However, as indicated in Fig. 7, the performance of the weight-based technique over places around the average temperature of the study area is getting poorer than the proposed technique. The SVD unmixing performs even poorer than the other two techniques with large variances in not only high temperature places, but also places with average temperature of the study area.

The color-coded scatterplots bring further information about the performances of the techniques over certain LULC types. Here, the major LULC types including residential, transportation, agricultural and forest areas are considered. The built-up areas are mainly clustered at the high temperature tails of the scatterplots, yet with exception of the pixels such as urban green areas classified as built-up areas and fall into the low temperature tails of the scatterplots. The largest proportion of pixels in the study area are agricultural with different types of crops, thus the temperature of these pixels varies from low to high. Forest and transportation areas are with small temperature variations and clustered at the lower and higher tails of the scatterplots, respectively. Due to the large number pixels classified as agricultural land, these pixels dominate the patterns of the scatterplots and thus impose major influence on the evaluation of the techniques.

The statistics of predictive capacities of all three techniques over the entire study area are summarized in Table 3. Corresponding to the observations in Fig. 7, all the techniques tend to overestimate the mean temperature of the study area. The weight-based and SVD unmixing- 
(a)

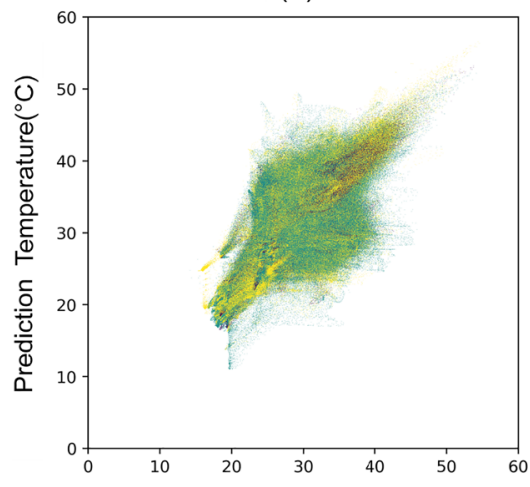

(b)

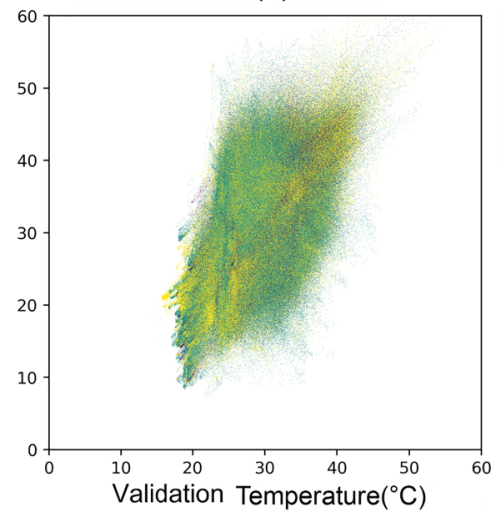

(c)

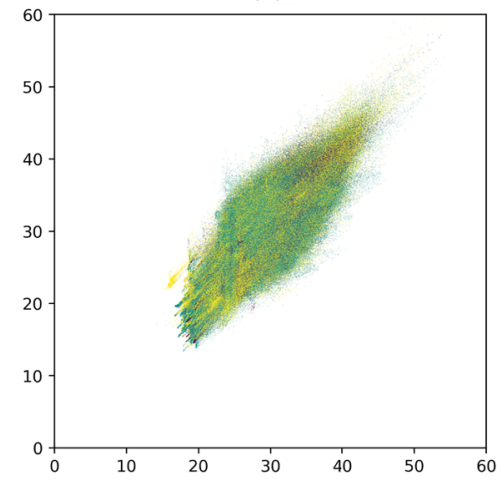

Residential Transportation Agricultural Forest Others

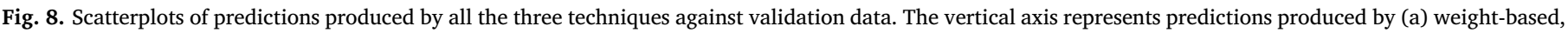
(b) SVD unmixing-based and (c) TC unmixing based techniques, while the validation data is represented by horizontal axis.

based bring more than $2{ }^{\circ} \mathrm{C}$ of overestimation, whereas the TC unmixing-based technique reduces such overestimation to $1.27{ }^{\circ} \mathrm{C}$. TC unmixing-based further reduces overestimation of high temperature and also underestimation of low temperature compared to the other two techniques. This is already visible in the large variance of predicted LST produced by the other two techniques as shown in Fig. 8. The combined result of reduced predicted LST variance along with lower overestimated mean in the TC unmixing-based prediction produces a $\mathrm{R}^{2}$ of 0.71 and intercept of $2.39{ }^{\circ} \mathrm{C}$, which supersedes the performances of other downscaling techniques (Table 3 ).

When looking into the LULC level within the study area, the performances of the techniques can be evaluated across LULC types within each technique, across techniques within each LULC type, as well as across techniques and LULC types in combination.

Within the techniques, the performances of all the techniques across different LULC types share a similar pattern, where the predictions over agricultural area are better than those over the other LULC types in terms of the $\mathrm{R}^{2}$ (Table 4). The TC unmixing-based technique results in the highest $\mathrm{R}^{2}$ of 0.73 in downscaling LST over agriculture land. The poorest performances of all the techniques are found over either residential or transportation area. The original SVD unmixing-based technique generates the lowest $\mathrm{R}^{2}$ of 0.28 and 0.29 over transportation and residential area. However, by referring back to the scatterplots in Fig. 8, the interpretation of the performances across different LULC needs to be conservative. The poor performances of all the techniques over residential and transportation areas may due to not only the high heterogeneity of built environment, but also the limited amount of data. On the one hand, as all downscaling techniques impose assumptions of similar thermal behavior among spatially adjacent pixels, the heterogeneity of the LULC indeed is affecting the accuracy of the predictions. On the other hand, the small data size in terms of the number of pixels over residential and transportation areas eliminates the evaluation of model performance and makes the $\mathrm{R}^{2}$ subject to extreme values. As the large data size over agricultural area dominates the pattern of the scatterplots, the performances of the techniques over agricultural area is very much similar to those over the entire study area. This can be observed by comparing $\mathrm{R}^{2}$ in Table 3 and 4 .

Within each LULC type, the proposed TC unmixing-based technique produces significantly better predictions than those of the other two techniques in terms of higher $\mathrm{R}^{2}$ and lower RMSE. Over LULC types with less data and larger heterogeneity, the advantage of applying the TC unmixing-based technique increases (see Table 4).

When comparing the $\mathrm{R}^{2}$ across all techniques and LULC types, it seems that the difference between the $\mathrm{R}^{2}$ produced over residential area and the one over agricultural area is larger in the weight-based prediction than the other predictions. As mentioned above, the difference may be due to land surface heterogeneity and data size. However, the smaller difference found in the unmixing-based techniques may be partially attributed to the nature of the subpixel level treatment of the thermal patterns, which strictly split temperature change to target land surface endmembers or components, and is robust to LULC heterogeneity. Even the performance of the SVD unmixing-based technique is the poorest, the performance seems quite stable and the $\mathrm{R}^{2}$ does not change abruptly across different LULC types.

\subsection{Nighttime prediction}

Diurnal LST analysis and surface thermal balance studies require LST information during both day and night. Although downscaling the nighttime LST is rarely found, it is necessary to take this opportunity to examine the potential of available downscaling techniques. However, it is hardly possible to verfify nighttime downscaling results with limited nighttime Landsat imagery data. With only one nighttime image on May 26th, 2017 even within a span of two years yet contaminated by clouds, comparison among the techniques could also suffer from considerable amount of LULC change apart from the cloud contamination.

A minor visual analysis is provided in Fig. 9, where limited variation could be observed in the nighttime coarse-fine input images (Fig. 9(a) and (b)). Such limited variation could be due to the cloud contamination as none of the techniques has found a proper way to split the temperature change information into fine resolution. Thus the predictions stays very much similar to the input fine resolution data and indistinguishable (Fig. 9(d), (e) and (f)). Again, similar to the situation in Fig. 7, the based time fine resolution pixels located at places with coarse resolution missing values are not masked for visual convenience but excluded for statistical analysis. The statistical distributions of the

Table 3

Daytime prediction statistics.

\begin{tabular}{|c|c|c|c|c|c|c|c|c|}
\hline & $\operatorname{mean}\left({ }^{\circ} \mathrm{C}\right)$ & $\min \left({ }^{\circ} \mathrm{C}\right)$ & $\max \left({ }^{\circ} \mathrm{C}\right)$ & R-squared & $\mathrm{p}$-value & Slope & Intercept $\left({ }^{\circ} \mathrm{C}\right)$ & $\operatorname{RMSE}\left({ }^{\circ} \mathrm{C}\right)$ \\
\hline Validation & 28.39 & 14.18 & 54.57 & 1 & 1 & 1 & 1 & 1 \\
\hline Weight-based prediction & 30.91 & 11.04 & 56.55 & 0.51 & 0.01 & 0.76 & 7.91 & 3.91 \\
\hline SVD unmixing-based prediction & 30.92 & 7.11 & 62.12 & 0.33 & 0.01 & 0.81 & 4.9 & 4.72 \\
\hline TC unmixing-based prediction & 29.66 & 12.22 & 61.54 & 0.71 & 0.008 & 0.91 & 2.39 & 2.51 \\
\hline
\end{tabular}


Table 4

Predictive accuracy based upon sample land types.

\begin{tabular}{|c|c|c|c|c|c|c|c|c|}
\hline & \multicolumn{2}{|l|}{ Residential } & \multicolumn{2}{|c|}{ Transportation } & \multicolumn{2}{|c|}{ Agricultural } & \multicolumn{2}{|l|}{ Forestry } \\
\hline & R-squared & $\operatorname{RMSE}\left({ }^{\circ} \mathrm{C}\right)$ & R-squred & $\operatorname{RMSE}\left({ }^{\circ} \mathrm{C}\right)$ & R-squred & $\operatorname{RMSE}\left({ }^{\circ} \mathrm{C}\right)$ & R-squred & $\operatorname{RMSE}\left({ }^{\circ} \mathrm{C}\right)$ \\
\hline Weight-based prediction & 0.42 & 3.57 & 0.48 & 4.05 & 0.54 & 4.12 & 0.49 & 3.72 \\
\hline SVD unmixing-based prediction & 0.29 & 4.91 & 0.28 & 4.67 & 0.33 & 4.61 & 0.29 & 4.62 \\
\hline TC unmixing-based prediction & 0.64 & 2.56 & 0.69 & 2.51 & 0.73 & 2.48 & 0.69 & 2.36 \\
\hline
\end{tabular}

(a)

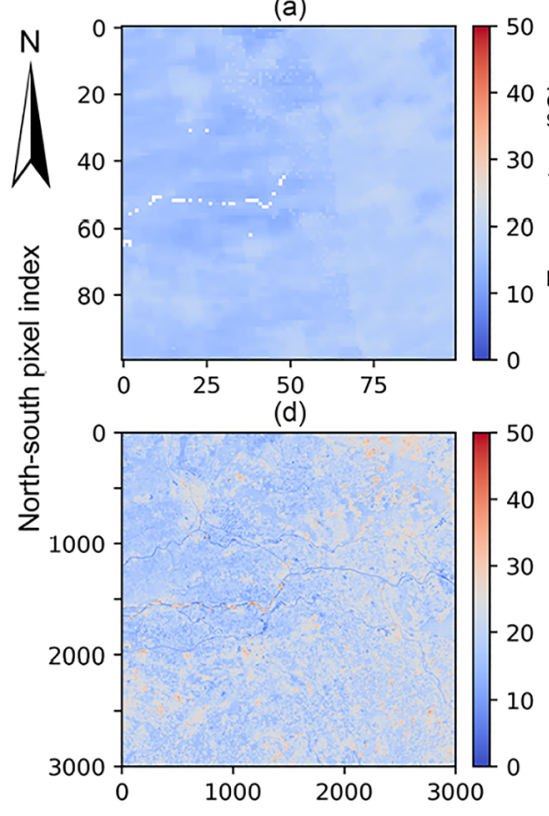

(b)
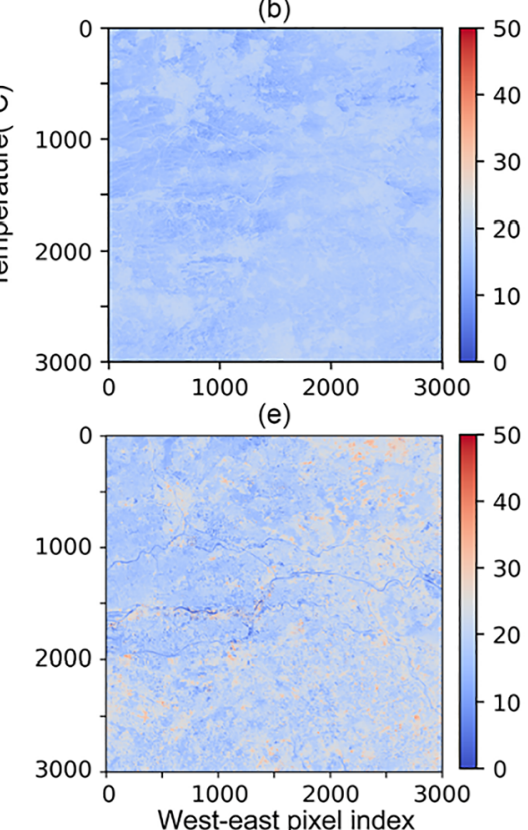

(c)

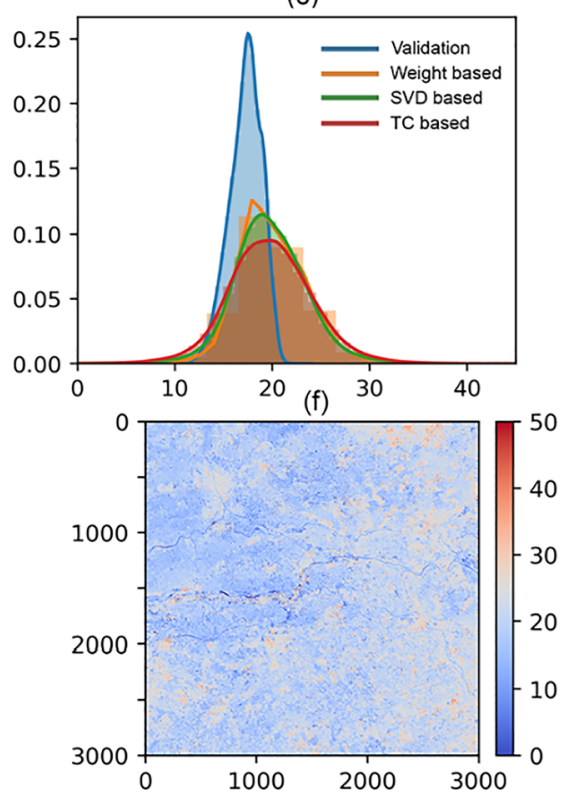

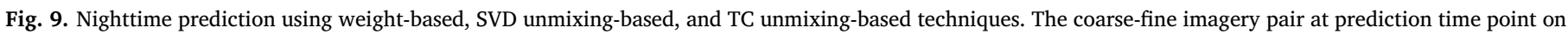

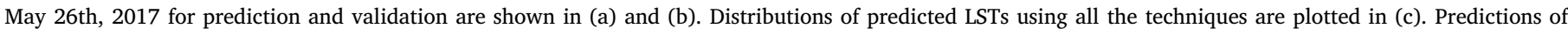
weight-based, SVD unmixing-based and TC unmixing-based techniques are visualized in (d), (e) and (f).

predictions further confirm that there is hardly any advantage in any of the techniques in the situation of poor quality input information. This section can also be treated as a test of the robustness of existing techniques against low quality data. More detailed information of nighttime downscaling results are summarized in Table 5.

\subsection{Outreach of proposed technique through sample application}

The downscaled LST forms a dense record of surface temperature dynamics and anomalies such as heatwaves and surface urban heat island (SUHI). In a zoomed-in area of Utrecht (Fig. 10(a)), the automatic weather station (AWS) air temperature measurement acquired at suburban Utrecht in De Bilt is used as background temperature. During the hottest month from July 11 to August 10 in 2018, the air temperature measured at the four time points of MODIS image acquirement reasonably reflect diurnal temperature change with daily high temperature at roughly $2 \mathrm{pm}$. The two heatwaves in 2018 are also observable with

Table 5

Nighttime prediction statistics.

\begin{tabular}{llll}
\hline & $\operatorname{mean}\left({ }^{\circ} \mathrm{C}\right)$ & $\min \left({ }^{\circ} \mathrm{C}\right)$ & $\max \left({ }^{\circ} \mathrm{C}\right)$ \\
\hline Validation & 17.26 & -1.59 & 23.79 \\
Weight-based prediction & 19.88 & -2.36 & 37.61 \\
SVD unmixing-based prediction & 19.79 & -5.56 & 41.57 \\
TC unmixing-based prediction & 19.18 & -6.68 & 42.92 \\
\hline
\end{tabular}

the first one starting on July 15 and lasting 13 days, and the second one starting on July 29th lasting 10 days. In general, both high and low resolution LSTs are higher than the background air temperature. Some exception is also shown due to precipitation forcing LST lower than the air temperature. Specifically, the LST within urban area recorded on a coarse pixel in MODIS image can be roughly $10{ }^{\circ} \mathrm{C}$ higher than background air temperature (Fig. 10(b)). Within this coarse urban pixel, the average LST of built environment (orange area) recorded by fine resolution pixel can be $7{ }^{\circ} \mathrm{C}$ higher than LST recorded in a coarse pixel in urban area. At the same time, the LST over non-built surface (vegetation and waterbody in green area) can be $4{ }^{\circ} \mathrm{C}$ cooler than the LST in corresponding coarse pixel. Such deviation of fine resolution LST from its corresponding coarse resolution LST highlights the capacity of our downscaling technique in splitting coarse LST information over multiple land surface types.

The SUHI is also obtained by choosing a reference rural coarse pixel, within which the fine resolution suburban LST is calculated as the average of vegetation pixels (Fig. 10(a)). The coarse resolution SUHI is obtained as the LST difference between the two reference coarse pixels, while the fine resolution SUHI is the difference between the average LST of the built environment and vegetation area within the coarse pixels. On average, the coarse resolution SUHI is larger than the fine resolution one as fine resolution image captures more variations in the LST. And this variation is successfully achieved by our downscaling technique. Due to the cloud contamination and many missing data points the SUHI time series, it is so far hard to conclude if the SUHIs obtained at the two resolutions is significantly different. Based on visual 
(a)
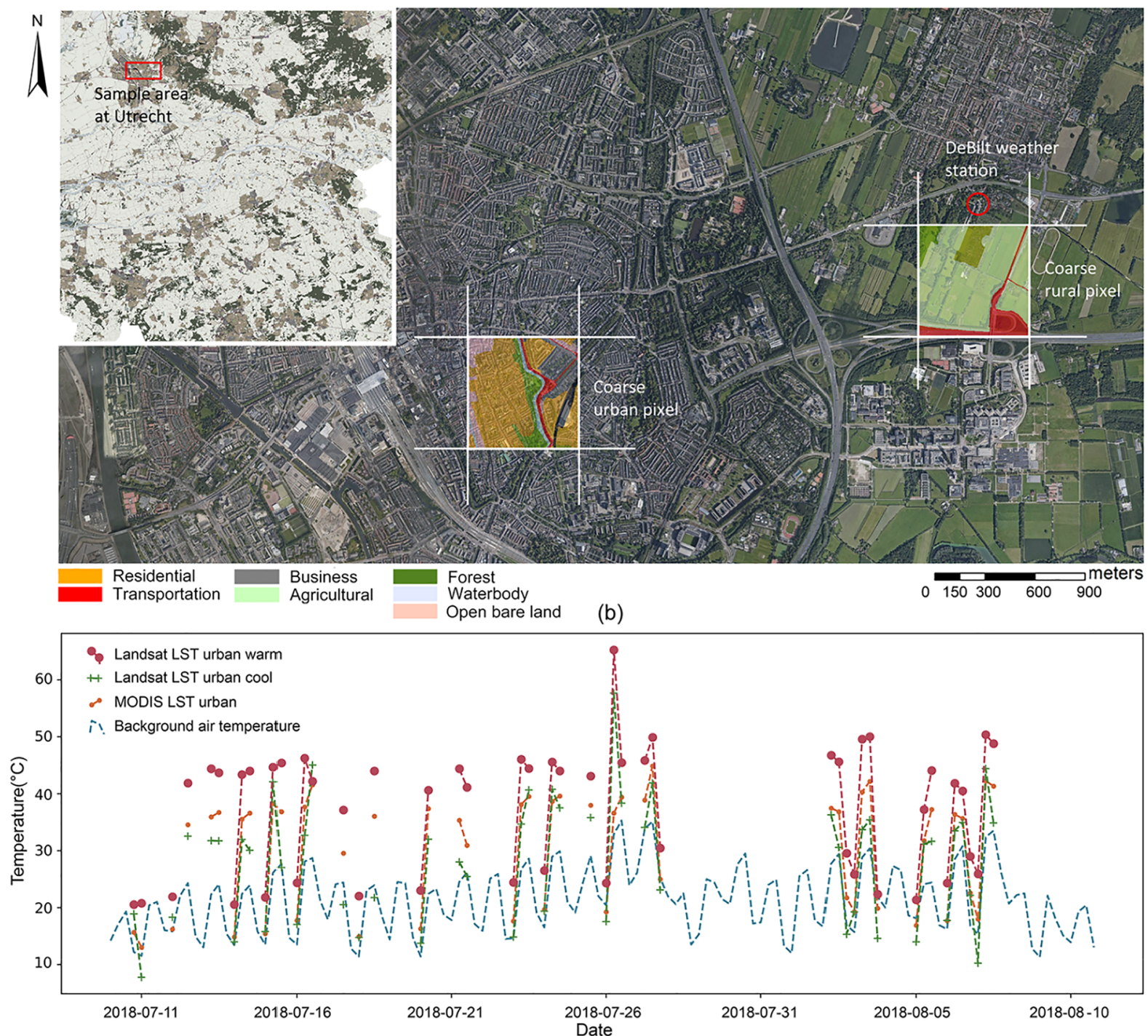

(c)

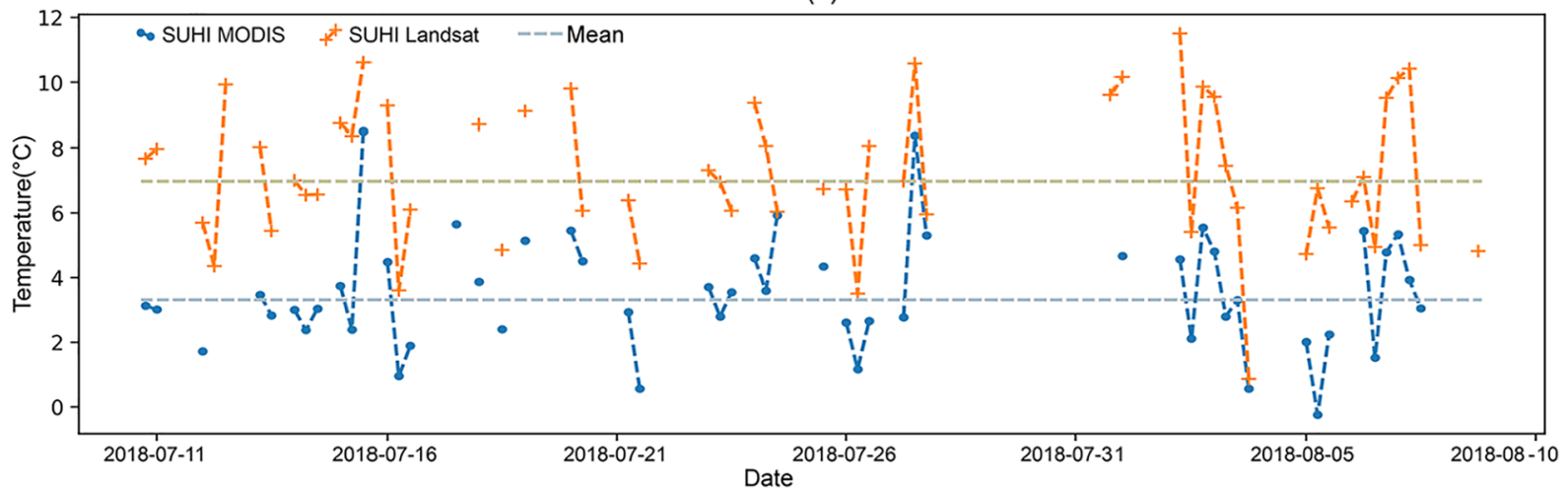

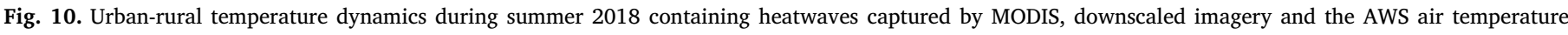

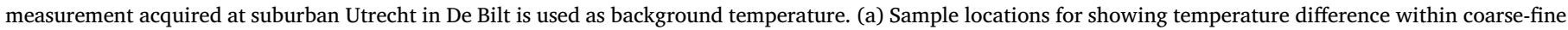

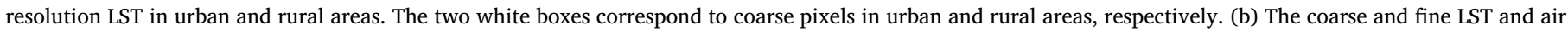

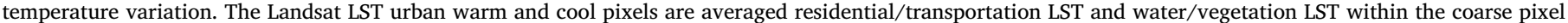

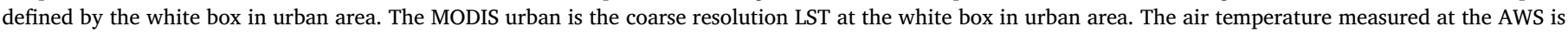

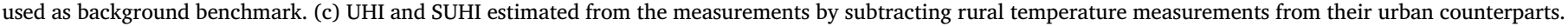


interpretation, the fine resolution SUHI reasonably reflects the temporal trend of SUHI recorded in the coarse pixels once reference pixels in urban and suburban areas are consistent at both coarse and fine resolution.

\section{Discussions}

Given the fact the unmixing-based downscaling techniques relies on auxiliary data and endmember information, improper selection of endmember by using thermally unsensitive land surface factors could easily devalue the use of extra data. This can be observed in the performance of the SVD unmixing-based technique, which is worse than the weight-based technique without using extra information other than the LST data. The proposed TC unmixing-based technique fulfill the purpose of characterizing both radiance and abundance change of land surface endmember in downscaling thermal images. The $20 \%$ improvements in the $\mathrm{R}^{2}$ renders the importance of using thermally meaningful components in splitting the LST change at the subpixel level. This improvement is reliable enough to assist the analysis in local scale environmental and microclimate studies.

Thus the feasibility and accuracy of spatiotemporal fusion downscaling is largely determined by the availability of properly selected land surface information to guide the splitting of coarse LST change information to fine resolution. Depending on the amount of predictor information required, spatiotemporal fusion downscaling techniques can be treated as predictor-intense techniques, predictor-free techniques or partial-predictor-dependent techniques. Regression-based downscaling referred in the introduction is a typical technique depending on LST sensitive land surface factors at both reference and prediction time points, which is less feasible to implement as land surface factors are not always available at each prediction time point. In contrast, the application of the weight-based technique to LST downscaling only stays within the LST datasets requiring no land surface predictors and makes bold assumption that LST pixels at both coarse and fine resolution may change similarly. Thus its application is restricted by heterogeneous land surfaces. The unmixing-based technique can be considered as partially depending on land surface factors as no further information about land surface is needed at prediction time. Then the question reduces to choosing proper land surface factors to guide the splitting of coarse LST into fine resolution. The results indicate that an improper choice or extraction of land surface factors leads to poor performance of downscaling as original land surface endmembers of soil, vegetation and dark surface are used. It is apparent that the reliability of downscaling is jointly determined by the choice of techniques along the predictor-intense and predictor-free spectrum, and choice of land surface factors (here LST sensitive), which in turn, is determined by data availability.

Besides technique selection and land surface factor extraction, almost all of the spatiotemporal fusion downscaling techniques are intrinsically restricted by the fact that LULC changes over time. Although our thermal component unmixing-based technique attempts to encompass both the radiance and thermal component fraction changes, the assumption only sticks to regular diurnal or seasonal changes. LST change caused by irregular LULC modifications would eliminate the reliability of downscaling. Thus there is always an assumption of fixed pattern either for land surface compositions or their change. Due to the frequent situation of limited data, regression-based techniques are not covered by this study and thus left out of discussion.

One last issue worth addressing is the necessity of the downscaling thermal imagery data to high resolution. Although high resolution information is intuitively preferable for detailed geographic pattern analysis, it also brings the issue of undesirable variations and noises. Thus the downscaling should always bring the research purpose in the consideration to avoid chasing high resolution aimlessly.

\section{Conclusions}

In summary, by hypothesizing the existence of thermal components (TCs) encompassing both radiance and abundance changes, this study developed a TC unmixing-based spatiotemporal fusion technique for LST downscaling with a factor of 30 . With this large factor of downscaling, the TC unmixing-based technique, compared to predictor-free weight-based and predictor-intense regression-based techniques, only requires an intermediate amount of LST sensitive land surface components, which are named as thermal components (TC) to guide the splitting of coarse LST into fine LST. The advantage of using TCs as opposed to the conventional SVD endmembers is that both endmember radiance and fraction changes are included in unmixing the LST change. It can even be flexible to encode either diurnal or seasonal change patterns depending on the way of extracting the TCs by using the NMF. The downscaled results supersede the classic weight-based and original SVD unmixing-based results, while the proposed technique does not require land surface predictors at each prediction time point for component extraction. Even though the TC unmixing-based technique performs poorer around built environment than it does over agricultural and forest areas, such short-fall also applies to the other benchmark techniques. At least, the downscaling results achieved a prediction accuracy over $60 \%$ over residential and transportation areas, which is still acceptable considering the large downscaling scale. However, it is still difficult to summarize the reason of the poor performance over built environment as the data size and LULC heterogeneity over the built environment may both influence the performance of the techniques over different LULC types. In practice, the proposed TC unmixing-based technique could capture spatial and temporal LST variations and anomaly, rendering its potential application in environment and climate studies at small scales. However, the TC unmixing-based technique still suffers from problems of over and underestimating the high and low temperature in the study area as other technique does. It is still so far difficult to assess its reliability of downscaling nighttime LST due to limited data. One of the major concern in the application of the proposed technique is the extraction of the TCs during a reasonable time window. Since the TCs seems to be highly related to the LULC composition and configuration, the extracted TCs by using input data across a large time span through the NMF may hard to be interpreted and falsely encode LST patterns. The prediction of LST across nearly a year at nighttime should also be considered with large uncertainty. In this study, the time span of the input data for TC extraction is around 3 months, where significant LULC change is negligible.

\section{Declaration of Competing Interest}

The authors declare that they have no known competing financial interests or personal relationships that could have appeared to influence the work reported in this paper.

\section{Acknowledgements}

This project was funded by The Global Geo Health Data Center (GGHDC), Utrecht University, the Netherlands.

\section{References}

Amiri, R., Weng, Q., Alimohammadi, A., Alavipanah, S.K., 2009. Spatial-temporal dynamics of land surface temperature in relation to fractional vegetation cover and land use/cover in the Tabriz urban area, Iran. Remote Sens. Environ. 113, 2606-2617.

Anderson, M., Norman, J., Kustas, W., Houborg, R., Starks, P., Agam, N., 2008. A thermalbased remote sensing technique for routine mapping of land-surface carbon, water and energy fluxes from field to regional scales. Remote Sens. Environ. 112, 4227-4241.

Azevedo, J., Chapman, L., Muller, C., 2016. Quantifying the daytime and night-time urban heat island in Birmingham, UK: A comparison of satellite derived land surface temperature and high resolution air temperature observations. Remote Sensing 8, 
153.

Barsi, J., Schott, J., Hook, S., Raqueno, N., Markham, B., Radocinski, R., 2014. Landsat-8 thermal infrared sensor (TIRS) vicarious radiometric calibration. Remote Sensing 6, 11607-11626.

Bechtel, B., Zakšek, K., Hoshyaripour, G., 2012. Downscaling land surface temperature in an urban area: A case study for Hamburg, Germany. Remote Sensing 4, 3184-3200.

Beniston, M., Diaz, H.F.J.G., 2004. The 2003 heat wave as an example of summers in a greenhouse climate? Observations and climate model simulations for Basel, Switzerland. Global Planetary Change 44, 73-81.

Cai, M., Ren, C., Xu, Y., Lau, K.K.-L., Wang, R., 2018. Investigating the relationship between local climate zone and land surface temperature using an improved WUDAPT methodology-A case study of Yangtze River Delta, China. Urban Clim. 24, 485-502.

Chase, T.N., Wolter, K., Pielke Sr., R.A., Rasool, I., 2006. Was the 2003 European summer heat wave unusual in a global context? Geophysical Research Letters 33.

Chen, B., Huang, B., Xu, B., 2015. Comparison of spatiotemporal fusion models: A review. Remote Sensing 7, 1798-1835.

Chen, X.-L., Zhao, H.-M., Li, P.-X., Yin, Z.-Y., 2006. Remote sensing image-based analysis of the relationship between urban heat island and land use/cover changes. Remote Sens. Environ. 104, 133-146.

Cho, K., Kim, Y., Kim, Y., 2018. Disaggregation of landsat-8 thermal data using guided SWIR Imagery on the scene of a wildfire. Remote Sensing 10.

Connors, J.P., Galletti, C.S., Chow, W.T., 2013. Landscape configuration and urban heat island effects: assessing the relationship between landscape characteristics and land surface temperature in Phoenix. Arizona 28, 271-283.

Coutts, A.M., Harris, R.J., Phan, T., Livesley, S.J., Williams, N.S., Tapper, N.J., 2016. Thermal infrared remote sensing of urban heat: Hotspots, vegetation, and an assessment of techniques for use in urban planning. Remote Sens. Environ. 186, 637-651.

Duan, S.B., Li, Z.L., 2016. Spatial downscaling of MODIS Land surface temperatures using geographically weighted regression: Case study in Northern China. IEEE Trans. Geosci. Remote Sens. 54, 6458-6469.

Duan, S.-B., Li, Z.-L., Li, H., Göttsche, F.-M., Wu, H., Zhao, W., Leng, P., Zhang, X., Coll, C., 2019. Validation of Collection 6 MODIS land surface temperature product using in situ measurements. Remote Sens. Environ. 225, 16-29.

Duan, S.-B., Li, Z.-L., Wu, H., Leng, P., Gao, M., Wang, C., 2018. Radiance-based validation of land surface temperature products derived from Collection 6 MODIS thermal infrared data. Int. J. Appl. Earth Observat. Geoinformat. 70, 84-92.

Estoque, R.C., Murayama, Y., 2015. Classification and change detection of built-up lands from Landsat-7 ETM + and Landsat-8 OLI/TIRS imageries: A comparative assessment of various spectral indices. Ecol. Ind. 56, 205-217.

Fensholt, R., Sandholt, I., 2003. Derivation of a shortwave infrared water stress index from MODIS near- and shortwave infrared data in a semiarid environment. Remote Sens. Environ. 87, 111-121.

Fu, P., Weng, Q., 2016. A time series analysis of urbanization induced land use and land cover change and its impact on land surface temperature with Landsat imagery. Remote Sens. Environ. 175, 205-214.

Gallo, K., McNab, A., Karl, T., Brown, J., Hood, J., Tarpley, J., 1993. The use of NOAA AVHRR data for assessment of the urban heat island effect. J. Appl. Meteorol. 32, 899-908.

Gallo, K.P., Tarpley, J.D., McNab, A.L., Karl, T.R., 1995. Assessment of urban heat islands: a satellite perspective. Atmos. Res. 37, 37-43.

Gao, F., Masek, J., Schwaller, M., Hall, F., 2006. On the blending of the Landsat and MODIS surface reflectance: Predicting daily Landsat surface reflectance. IEEE Trans. Geosci. Remote Sens. 44, 2207-2218.

Gevaert, C.M., García-Haro, F.J., 2015. A comparison of STARFM and an unmixing-based algorithm for Landsat and MODIS data fusion. Remote Sens. Environ. 156, 34-44.

Guo, G., Wu, Z., Xiao, R., Chen, Y., Liu, X., Zhang, X., 2015. Impacts of urban biophysical composition on land surface temperature in urban heat island clusters. Landscape Urban Plann. 135, 1-10.

Huang, B., Wang, J., Song, H., Fu, D., Wong, K., 2013. Generating high spatiotemporal resolution land surface temperature for urban heat island monitoring. IEEE Geosci. Remote Sensing Lett. 10, 1011-1015.

Jenerette, G.D., Harlan, S.L., Buyantuev, A., Stefanov, W.L., Declet-Barreto, J., Ruddell, B.L., Myint, S.W., Kaplan, S., Li, X., 2016. Micro-scale urban surface temperatures are related to land-cover features and residential heat related health impacts in Phoenix, AZ USA. Landscape Ecol. 31, 745-760.

Jiménez-Muñoz, J.C., Sobrino, J.A., Skoković, D., Mattar, C., Cristóbal, J., 2014. Land surface temperature retrieval methods from Landsat-8 thermal infrared sensor data. IEEE GeoscI. Remote Sens. Lett. 11, 1840-1843.

Kalma, J.D., McVicar, T.R., McCabe, M.F., 2008. Estimating land surface evaporation: A review of methods using remotely sensed surface temperature data. Surv. Geophys. 29, 421-469.

Kalnay, E., Cai, M., 2003. Impact of urbanization and land-use change on climate. Nature 423, 528-531.

Koc, C.B., Osmond, P., Peters, A., Irger, M., 2018. Understanding land surface temperature differences of local climate zones based on airborne remote sensing data. IEEE J. Sel. Top. Appl. Earth Observat. Remote Sens. 11, 2724-2730.

Kuang, W., Dou, Y., Zhang, C., Chi, W., Liu, A., Liu, Y., Zhang, R., Liu, J., 2015. Quantifying the heat flux regulation of metropolitan land use/land cover components by coupling remote sensing modeling with in situ measurement. J. Geophys. Res. Atmospheres 120, 113-130.

Kustas, W.P., Nieto, H., Morillas, L., Anderson, M.C., Alfieri, J.G., Hipps, L.E., Villagarcía, L., Domingo, F., Garcia, M., 2016. Revisiting the paper "Using radiometric surface temperature for surface energy flux estimation in Mediterranean drylands from a two-source perspective. Remote Sens. Environ. 184, 645-653.

Lee, D.D., Seung, H.S., 1999. Learning the parts of objects by non-negative matrix factorization. Nature 401, 788-791.

Lee, D.D., Seung, H.S., 2001. Algorithms for non-negative matrix factorization. In: Advances in Neural Information Processing Systems, pp. 556-562.

Li, F., Kustas, W.P., Anderson, M.C., Prueger, J.H., Scott, R.L., 2008. Effect of remote sensing spatial resolution on interpreting tower-based flux observations. Remote Sens. Environ. 112, 337-349.

Li, Z.-L., Tang, B.-H., Wu, H., Ren, H., Yan, G., Wan, Z., Trigo, I.F., Sobrino, J.A., 2013. Satellite-derived land surface temperature: Current status and perspectives. Remote Sens. Environ. 131, 14-37.

Ma, J., Zhang, W., Marinoni, A., Gao, L., Zhang, B., 2018. An improved spatial and temporal reflectance unmixing model to synthesize time series of landsat-like images. Remote Sens. 10, 1388.

Miao, L.D., Qi, H.R., 2007. Endmember extraction from highly mixed data using minimum volume constrained nonnegative matrix factorization. IEEE Trans. Geosci. Remote Sens. 45, 765-777.

Mira, M., Ninyerola, M., Batalla, M., Pesquer, L., Pons, X., 2017. Improving mean minimum and maximum month-to-month air temperature surfaces using satellitederived land surface temperature. Remote Sensing 9, 1313.

Mirzaei, P.A., Society, 2015. Recent challenges in modeling of urban heat island. Sustainable Cities Soc. 19, 200-206.

Oyler, J.W., Dobrowski, S.Z., Holden, Z.A., Running, S.W., 2016. Remotely sensed land skin temperature as a spatial predictor of air temperature across the conterminous United States. J. Appl. Meteorol. Climatol. 55, 1441-1457.

Peng, Y.D., Li, W.S., Luo, X.B., Li, H., 2019. A Geographically and temporally weighted regression model for spatial downscaling of MODIS Land surface temperatures over urban heterogeneous regions. IEEE Trans. Geosci. Remote Sens. 57, 5012-5027.

Pu, R., Gong, P., Michishita, R., Sasagawa, T., 2006. Assessment of multi-resolution and multi-sensor data for urban surface temperature retrieval. Remote Sens. Environ. 104, 211-225

Quan, J., Zhan, W., Ma, T., Du, Y., Guo, Z., Qin, B., 2018. An integrated model for generating hourly Landsat-like land surface temperatures over heterogeneous landscapes. Remote Sens. Environ. 206, 403-423.

Quattrochi, D.A., Luvall, J.C., 1999. Thermal infrared remote sensing for analysis of landscape ecological processes: methods and applications. Landscape Ecol. 14, 577-598.

Rajasekar, U., Weng, Q., 2009. Urban heat island monitoring and analysis using a nonparametric model: A case study of Indianapolis. Isprs J. Photogramm. Remote Sens. 64, 86-96.

Rebetez, M., Dupont, O., Giroud, M., 2009. An analysis of the July 2006 heatwave extent in Europe compared to the record year of 2003. Theoret. Appl. Climatol. 95, 1-7.

Sandholt, I., Rasmussen, K., Andersen, J., 2002. A simple interpretation of the surface temperature/vegetation index space for assessment of surface moisture status. Remote Sens. Environ. 79, 213-224.

Schwarz, N., Schlink, U., Franck, U., Großmann, K., 2012. Relationship of land surface and air temperatures and its implications for quantifying urban heat island indicators-An application for the city of Leipzig (Germany). Ecol. Ind. 18, 693-704.

Shen, H., Huang, L., Zhang, L., Wu, P., Zeng, C., 2016a. Long-term and fine-scale satellite monitoring of the urban heat island effect by the fusion of multi-temporal and multisensor remote sensed data: A 26-year case study of the city of Wuhan in, China. Remote Sens. Environ. 172, 109-125.

Shen, H., Meng, X., Zhang, L., 2016b. An integrated framework for the spatio-temporal-spectral fusion of remote sensing images. IEEE Trans. Geosci. Remote Sens. 54, 7135-7148.

Shen, H.F., Wu, P.H., Liu, Y.L., Ai, T.H., Wang, Y., Liu, X.P., 2013. A spatial and temporal reflectance fusion model considering sensor observation differences. Int. J. Remote Sens. 34, 4367-4383.

Small, C., 2003. High spatial resolution spectral mixture analysis of urban reflectance. Remote Sens. Environ. 88, 170-186.

Small, C., 2004. The Landsat ETM + spectral mixing space. Remote Sens. Environ. 93 $1-17$.

Sobrino, J., Oltra-Carrió, R., Sòria, G., Bianchi, R., Paganini, M., 2012. Impact of spatial resolution and satellite overpass time on evaluation of the surface urban heat island effects. Remote Sens. Environ. 117, 50-56.

Steven, M.D., Malthus, T.J., Baret, F., Xu, H., Chopping, M.J., 2003. Intercalibration of vegetation indices from different sensor systems. Remote Sens. Environ. 88, 412-422.

Stoll, M.J., Brazel, A.J., 1992. Surface-air temperature relationships in the urban environment of Phoenix, Arizona. Phys. Geography 13, 160-179.

Tan, Z., Yue, P., Di, L., Tang, J., 2018. Deriving high spatiotemporal remote sensing images using deep convolutional network. Remote Sens. 10, 1066.

Tucker, C.J., 1980. Remote-sensing of leaf water-content in the near-infrared. Remote Sens. Environ. 10, 23-32.

Voogt, J.A., Oke, T.R., 2003. Thermal remote sensing of urban climates. Remote Sens. Environ. 86, 370-384.

Wan, Z., 2014. New refinements and validation of the collection-6 MODIS land-surface temperature/emissivity product. Remote Sens. Environ. 140, 36-45.

Wang, F., Qin, Z., Song, C., Tu, L., Karnieli, A., Zhao, S., 2015. An improved monowindow algorithm for land surface temperature retrieval from Landsat 8 thermal infrared sensor data. Remote Sens. 7, 4268-4289.

Weng, Q., Firozjaei, M.K., Kiavarz, M., Alavipanah, S.K., Hamzeh, S., 2019. Normalizing land surface temperature for environmental parameters in mountainous and urban 
areas of a cold semi-arid climate. Sci. Total Environ. 650, 515-529.

Weng, Q., Fu, P., Gao, F., 2014. Generating daily land surface temperature at Landsat resolution by fusing Landsat and MODIS data. Remote Sens. Environ. 145, 55-67.

Wu, H., Ye, L.-P., Shi, W.-Z., Clarke, K.C., 2014. Assessing the effects of land use spatial structure on urban heat islands using HJ-1B remote sensing imagery in Wuhan, China. Int. J. Appl. Earth Observat. Geoinformat. 32, 67-78.

Zhang, K., Kimball, J.S., Running, S.W., 2016. A review of remote sensing based actual evapotranspiration estimation. Wiley Interdiscip. Reviews: Water 3, 834-853.

Zhou, D.C., Zhao, S.Q., Liu, S.G., Zhang, L.X., Zhu, C., 2014. Surface urban heat island in China's 32 major cities: Spatial patterns and drivers. Remote Sens. Environ. 152, 51-61.

Zhu, X., Cai, F., Tian, J., Williams, T., 2018. Spatiotemporal fusion of multisource remote sensing data: literature survey, taxonomy, principles, applications, and future directions. Remote Sensing 10, 527. 